\title{
Chemical evolution of the Galactic bulge as traced by microlensed dwarf and subgiant stars ${ }^{\star, \star \star}$
}

\section{Ages, metallicities, detailed elemental abundances, and connections to the Galactic thick disc}

\author{
T. Bensby ${ }^{1}$, S. Feltzing ${ }^{2}$, J.A. Johnson ${ }^{3}$, A. Gould ${ }^{3}$, D. Adén ${ }^{2}$, M. Asplund ${ }^{4}$, J. Meléndez ${ }^{5}$, A. Gal-Yam ${ }^{6}$, \\ S. Lucatello ${ }^{7}$, H. Sana ${ }^{1,8}$, T. Sumi ${ }^{9}$, N. Miyake ${ }^{9}$, D. Suzuki ${ }^{9}$, C. Han $^{10}$, I. Bond ${ }^{11}$, and A. Udalski ${ }^{12}$ \\ ${ }^{1}$ European Southern Observatory, Alonso de Cordova 3107, Vitacura, Casilla 19001, Santiago 19, Chile \\ e-mail: tbensby@eso.org \\ 2 Lund Observatory, Box 43, 22100 Lund, Sweden \\ 3 Department of Astronomy, Ohio State University, 140 W. 18th Avenue, Columbus, OH 43210, USA \\ ${ }^{4}$ Max Planck Institute for Astrophysik, Garching, Germany \\ 5 Centro de Astrofísica, Universidade do Porto, Rua das Estrelas, 4150-762 Porto, Portugal \\ ${ }^{6}$ Benoziyo Center for Astrophysics, Weizmann Institute of Science, 76100 Rehovot, Israel \\ INAF-Astronomical Observatory of Padova, Vicolo dell'Osservatorio 5, 35122 Padova, Italy \\ 8 Universiteit van Amsterdam, Sterrenkundig Instituut "Anton Pannekoek", Postbus 94249, 1090 GE Amsterdam, The Netherlands \\ 9 Solar-Terrestrial Enivironment Laboratory, Nagoya University, Furo-cho, Chikusa-ku, Nagoya 464-8601, Japan \\ 10 Department of Physics, Chungbuk National University, Cheongju 361-763, Republic of Korea \\ 11 Institute of Information and Mathematical Sciences, Massey University, Albany Campus, Private Bag 102-904, \\ North Shore Mail Centre, Auckland, New Zealand \\ 12 Warsaw University Observatory, A1. Ujazdowskie 4, 00-478 Warszawa, Poland
}

Received 26 November 2009 / Accepted 6 January 2010

\begin{abstract}
Context. The Bulge is the least understood major stellar population of the Milky Way. Most of what we know about the formation and evolution of the Bulge comes from bright giant stars. The underlying assumption that giants represent all the stars, and accurately trace the chemical evolution of a stellar population, is under debate. In particular, recent observations of a few microlensed dwarf stars give a very different picture of the evolution of the Bulge from that given by the giant stars.

Aims. We aim to resolve the apparent discrepancy between Bulge metallicity distributions derived from microlensed dwarf stars and giant stars. Additionally, we aim to put observational constraints on the elemental abundance trends and chemical evolution of the Bulge.

Methods. We perform a detailed elemental abundance analysis of dwarf stars in the Galactic bulge, based on high-resolution spectra that were obtained while the stars were optically magnified during gravitational microlensing events. The analysis method is the same as for a large sample of $\mathrm{F}$ and $\mathrm{G}$ dwarf stars in the Solar neighbourhood, enabling a fully differential comparison between the Bulge and the local stellar populations in the Galactic disc.

Results. We present detailed elemental abundances and stellar ages for six new dwarf stars in the Galactic bulge. Combining these with previous events, here re-analysed with the same methods, we study a homogeneous sample of 15 stars, which constitute the largest sample to date of microlensed dwarf stars in the Galactic bulge. We find that the stars span the full range of metallicities from $[\mathrm{Fe} / \mathrm{H}]=-0.72$ to +0.54 , and an average metallicity of $\langle[\mathrm{Fe} / \mathrm{H}]\rangle=-0.08 \pm 0.47$, close to the average metallicity based on giant stars in the Bulge. Furthermore, the stars follow well-defined abundance trends, that for $[\mathrm{Fe} / \mathrm{H}]<0$ are very similar to those of the local Galactic thick disc. This suggests that the Bulge and the thick disc have had, at least partially, comparable chemical histories. At sub-solar metallicities we find the Bulge dwarf stars to have consistently old ages, while at super-solar metallicities we find a wide range of ages. Using the new age and abundance results from the microlensed dwarf stars we investigate possible formation scenarios for the Bulge.
\end{abstract}

Key words. gravitational lensing: micro - Galaxy: bulge - Galaxy: formation - Galaxy: evolution - Galaxy: disk - stars: abundances

* Based on observations made with the European Southern Observatory telescopes, Program IDs 082.B-0453 and 083.B-0265.

$\star \star$ Table 5 is also available in electronic form at the CDS and full Table 4 is only available in electronic form at the CDS via anonymous ftp to cdsarc.u-strasbg. fr $(130.79 .128 .5)$ or via http://cdsweb.u-strasbg.fr/cgi-bin/qcat?]/A+A/512/A41

\section{Introduction}

The Galactic bulge is a major stellar component of the Milky Way. Estimations of its mass range from 10\% (Oort 1977) to $25 \%$ (Sofue et al. 2009) of the total stellar mass of the Galaxy. It is a peanut shaped barred bulge and occupies the inner $\sim 1 \mathrm{kpc}$ of the Galaxy (Frogel 1988). Recent results for the Bulge shape 
give a scale length for the bar major axis of $\sim 1.5 \mathrm{kpc}$ (Rattenbury et al. 2007). The kinematic properties of the Bulge are intermediate between a rotationally supported system and a velocity dispersion dominated system (e.g., Minniti \& Zoccali 2008). The markedly different stellar populations that inhabit this region of the Galaxy make it important to discern the formation and evolution as part of the understanding of the overall formation of the Galaxy. In addition, bulges are common features among luminous galaxies, and determining the origin of the Bulge and the corresponding observational signatures (e.g., boxy isophotes, high $[\alpha / \mathrm{Fe}]$ ratios, no age dispersion) are important steps in decoding the formation of bulges in general.

The differences among the Bulge, the thin and thick discs in the Solar neighbourhood, and the Galactic stellar halo have been discovered and detailed through extensive photometric and spectroscopic observations of stars. Red giants are generally the only stars bright enough for high-resolution spectroscopy at the distance of the Bulge, and they have been intensively studied in the optical and infrared, particularly in a few fields including Baade's window (e.g. McWilliam \& Rich 1994; Fulbright et al. 2006; Zoccali et al. 2008; Meléndez et al. 2008; Cunha \& Smith 2006; Ryde et al. 2009). Chemically, the Bulge is decidedly more metal-rich than the stellar halo, with the mean $[\mathrm{Fe} / \mathrm{H}]$ from the Zoccali et al. (2008) sample slightly below solar metallicity, and a possible vertical metallicity gradient of 0.6 dex per kpc, although a comparison with $\mathrm{M}$ giants in the inner Bulge may indicate a flattening of the gradient in the central regions (Rich et al. 2007). The abundance ratios show enhanced $[\alpha / \mathrm{Fe}]$ ratios that persist to higher $[\mathrm{Fe} / \mathrm{H}]$ than in local thin disc stars, but show good agreement with thick disc giants (Meléndez et al. 2008; Alves-Brito et al. 2010). Furthermore, the stars at the Bulge main-sequence turnoff are red, and isochrone fitting has shown that the majority of the stars in the Bulge are old (e.g., Holtzman et al. 1993; Feltzing \& Gilmore 2000; Zoccali et al. 2003; Clarkson et al. 2008).

These observations have fuelled an intense debate on the origin of the Bulge. The established concordance model of cosmology provides the framework to understand the formation of the Milky Way by hierarchical merging, but the formation of the components of the Galaxy requires important physics, such as star formation and feedback, that is unresolved and parametrised in current models. However, there are two basic scenarios by which a bulge is built up in simulations (see Rahimi et al. 2010, and references therein). The first is by mergers, where subclumps coming together in the early phases of Galactic evolution combine to form the Bulge out of both accreted stars and stars formed in situ. The second is secular evolution, where the Bulge is created gradually out of the Galactic disc (e.g., Kormendy \& Kennicutt 2004). The merger model is favoured by the metallicity gradient, while the secular evolution model is favored by cylindrical rotation and by agreement (in terms of mean metallicity abundance trends, and ages) with the Galactic thick disc (e.g., Howard et al. 2009).

As mentioned above, spectroscopic observations of stars in the Bulge have usually been confined to giants. This limits our knowledge of the Bulge in several ways. First, because much of our knowledge of the Solar neighbourhood relies on dwarf stars (e.g. Edvardsson et al. 1993), any systematic offsets between the metallicity scale of giant and dwarf stars is cause for concern. In this context, Taylor \& Croxall (2005) have shown that there is a lack of very metal-rich $([\mathrm{Fe} / \mathrm{H}]>+0.2)$ giants in the Solar neighbourhood and that the mean metallicity of local giants is lower than for dwarfs. Recent studies of nearby giants (Luck \& Heiter 2007; Takeda et al. 2008) confirm the lack of very metal-rich stars. Santos et al. (2009) suggest that there may be systematic errors in the metallicity determinations of metal-rich giants. Secondly, there are several pieces of evidence that giant stars may not accurately represent all the stars. For the metal-rich cluster NGC 6791, Kalirai et al. (2007) proposed that the explanation for the large number of low-mass He white dwarfs was that about $40 \%$ of the stars do not become red clump stars, skipping entire phases of stellar evolution altogether because of high mass loss. Kilic et al. (2007) proposed a similar mechanism to explain the non-binary He white dwarfs found in the Solar neighbourhood. Finally, with turnoff stars, ages can be determined for individual stars, and an age-metallicity relationship derived, which is not possible for giant stars. This means that a true study of the Bulge requires the study of dwarf stars. However, at the distance of the Bulge, dwarf stars are too faint for abundance analyses based on high-resolution spectra. Turnoff stars in the Bulge have V magnitudes around 19 to 20 (compare, e.g., the colour-magnitude diagrams in Feltzing \& Gilmore 2000). However, in the event that a Bulge dwarf star is lensed by a foreground object, the magnitude of the star can increase by more than 5 magnitudes, in which case a high-resolution spectrum can be obtained and the star analysed in a similar manner as the dwarf stars in the Solar neighbourhood.

There were several spectroscopic observations of microlensed Bulge stars in the 1990s, but the first high-resolution spectrum of a dwarf star was presented in Minniti et al. (1998). Complete high-resolution spectroscopic abundance analyses have been published for eight microlensing events toward the Bulge (Johnson et al. 2007, 2008; Cohen et al. 2008, 2009; Bensby et al. 2009a,b; Epstein et al. 2010). Initially, it appeared that the microlensed Bulge dwarf stars were typically much more metal-rich than the giants in the Bulge, and Epstein et al. (2010) found, using a Kolmogorov-Smirnow (KS) test, a very low probability of only $1.6 \%$ that these eight microlensed dwarf stars in the Bulge were drawn from the same metallicity distribution (MDF) as the sample of Bulge giants from Zoccali et al. (2008). Cohen et al. (2008) proposed that a similar mechanism to the one occurring in NGC 6791 was occurring in the Bulge, preventing the most metal-rich stars from being included in the giant surveys. Arguments against this idea presented by Zoccali et al. (2008) were based on the luminosity function along the main sequence and red giant branch, showing no lack of RGB stars, with respect to the prediction of theoretical models.

In addition, the microlensed Bulge dwarf stars showed good agreement in abundance ratios with the thick disc stars in the Solar neighbourhood (Bensby et al. 2009a,b). However, comparisons have been hampered because of the small number of microlensed stars that did not always cover the $[\mathrm{Fe} / \mathrm{H}]$ range of interest. Also, individual age estimates were provided for stars near the turnoff subgiant branch, including some stars that could be younger than the canonical old Bulge population (Johnson et al. 2008; Bensby et al. 2009b).

We will here present detailed elemental abundance results for six new microlensing events toward the Galactic bulge. We also re-analyse the events previously studied by Cavallo et al. (2003); Cohen et al. (2009) and Epstein et al. (2010). Combining these data with the results from Bensby et al. (2009a) and Bensby et al. (2009b) (which includes a re-analysis of the events from Johnson et al. 2007, 2008; Cohen et al. 2008) we now have a sample of 15 microlensed dwarf stars in the Bulge that have been homogeneously analysed using the exact same methods. 
T. Bensby et al.: Chemical evolution of the Galactic bulge as traced by microlensed dwarf and subgiant stars. II.
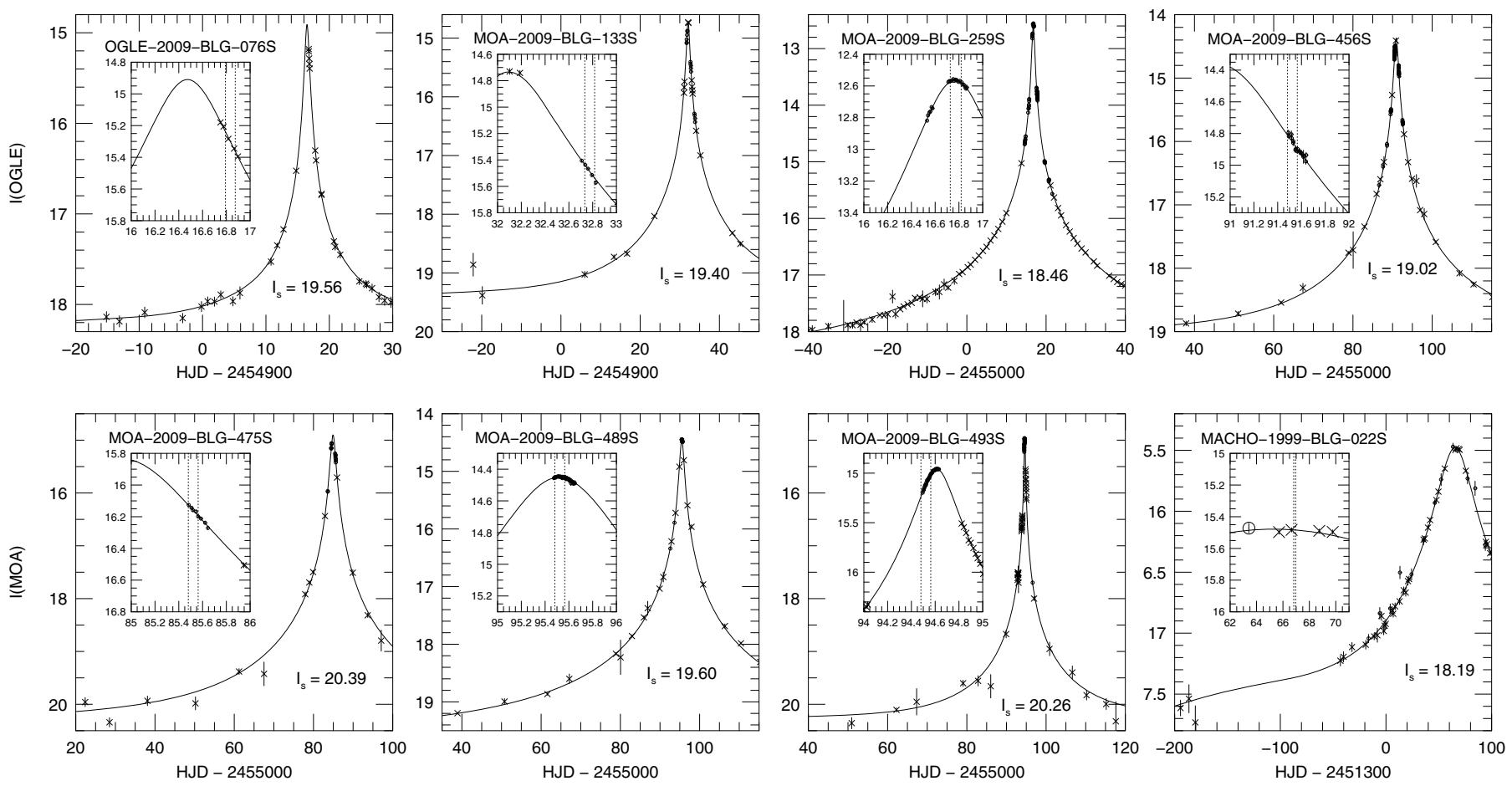

Fig. 1. Light curves for the eight new microlensing events. The photometry comes from the surveys indicated by their names (MOA or OGLE), except for MACHO-1999-BLG-022S that has data from from both OGLE (circles) and binned MACHO data (crosses). Each plot has a zoom window, showing the time intervals when the source stars were observed with high-resolution spectrographs. In each plot the un-lensed magnitude of the source star is also given $\left(I_{\mathrm{S}}\right)$.

\section{Observations and data reduction}

In order to trigger observations of these highly magnified stars we rely on the OGLE ${ }^{1}$ and $\mathrm{MOA}^{2}$ projects that every night monitor about 100 million stars toward the Bulge to detect variations in their brightnesses. If an object shows a well-defined rise in brightness, a microlensing alert is announced. Every year, 600-800 events are detected. Based on the photometric data obtained by the MOA and OGLE surveys it is possible to model the event, and make predictions of the length of the event, peak brightness, and time of peak brightness. Stars are identified as likely dwarf stars based on their unlensed magnitudes and colour differences relative to the red clump stars. This is done in instrumental magnitudes. The majority are low magnification events, and only a few have unlensed brightnesses of $V=18-20$, characteristic of dwarf stars in the Bulge at a distance of $\sim 8 \mathrm{kpc}$. During a regular Bulge season in the Southern hemisphere, typically around 10 high-magnification events of dwarf stars in the Bulge are detected ${ }^{3}$. To catch these unpredictable events, we have an ongoing Target of Opportunity (ToO) program at the ESO Very Large Telescope on Paranal in Chile. Observations can then be triggered with only a few hours notice.

\footnotetext{
${ }^{1}$ OGLE is short for Optical Gravitational Lens Experiment, http://ogle. astrouw. edu.pl (Udalski 2003).

2 MOA is short for Microlensing Observations for Astrophysics, http://www . phys . canterbury. ac.nz/moa (e.g., Bond et al. 2001).

3 With the new OGLE-IV camera that will be in operation in the first half of 2010 the field-of-view will increase from the 0.3 square degrees of OGLE-III to 1.4 square degrees, resulting in a substantial increase in the number of detected microlensing events.
}

\subsection{The 2009 events observed with UVES}

On March 21, 2009, the OGLE early warning system identified OGLE-2009-BLG-076 to be a possible high-magnification microlensing event toward the Galactic bulge. As the magnitude of the source star before the microlensing event indicated that it was either a dwarf or a subgiant star, we triggered observations with the UVES spectrograph (Dekker et al. 2000) located on UT2 at the ESO Very Large Telescope on Paranal. Due to the limited visibility of the Bulge in March, the target had to be observed towards the end of the night. Hence, OGLE-2009-BLG-076S was observed on March 26, a few hours after reaching peak brightness (see Fig. 1). A few weeks later, on April 11, we observed the MOA-2009-BLG-133, this time first alerted by the MOA collaboration, also with UVES. The third event was observed in the beginning of July, MOA-2009-BLG-259S. This object was observed during the UVES red arm upgrade, so we could only obtain a spectrum with the blue CCD that has a limited wavelength coverage of 3700-5000 ̊. In September, the end of ESO observing period P83, we saw an explosion of microlensing events and another four source stars were observed with UVES: MOA2009-BLG-475S on Sep. 10, MOA-2009-BLG-456S on Sep. 16, MOA-2009-BLG-493S on Sep. 19, and MOA-2009-BLG-489S on Sep. 20. The UVES red arm was now back with two new red CCDs with increased sensitivities.

For the 2009 observations with UVES listed above, each target was observed for a total of two hours, split into either four $30 \mathrm{~min}$ or three $40 \mathrm{~min}$ exposures. Using UVES with dichroic number 2, each observation resulted in spectra

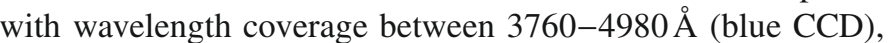
5680-7500 $\AA$ (lower red CCD), and 7660-9460 (upper red 
Table 1. Summary ${ }^{\dagger}$ of the, so far, 15 dwarf star microlensing events in the Bulge that have been observed with high-resolution spectrographs. They have been sorted according to their metallicities (as given in Table 2).

\begin{tabular}{|c|c|c|c|c|c|c|c|c|c|c|c|c|c|}
\hline Object & $\begin{array}{l}\text { RAJ2000 } \\
\text { [hh:mm:ss] }\end{array}$ & $\begin{array}{c}\text { DEJ2000 } \\
\text { [dd:mm:ss] }\end{array}$ & $\begin{array}{c}l \\
{[\mathrm{deg}]}\end{array}$ & $\begin{array}{c}b \\
{[\mathrm{deg}]}\end{array}$ & $\begin{array}{c}T_{\mathrm{E}} \\
\text { [days] }\end{array}$ & $\begin{array}{r}T_{\max } \\
\text { [HJD] }\end{array}$ & $A_{\max }$ & $\begin{array}{c}T_{\text {obs }} \\
{[\mathrm{MJD}]}\end{array}$ & $\begin{array}{l}\text { Exp. } \\
{[\mathrm{s}]}\end{array}$ & & s & $R$ & Ref. \\
\hline OGLE-2009 & $17: 58: 31.9$ & $-29: 12: 17.8$ & +1.21 & -2.56 & 36.9 & 4916.46 & 70 & 4916.291 & 7200 & 30 & UVES & 45000 & B09a \\
\hline MOA-2009-BLG-493S & $17: 55: 46.0$ & $-28: 48: 25.8$ & +1.25 & -1.84 & 13.2 & 5094.61 & 150 & 5093.980 & 7200 & 40 & UVES & 45000 & TW \\
\hline MOA-2009-BLG-133S & $18: 06: 32.8$ & $-31: 30: 10.7$ & +0.05 & -5.19 & 26.6 & 4932.10 & 70 & 4932.233 & 7200 & 35 & UVES & 45000 & TW \\
\hline MOA-2009-BLG-475S & $18: 02: 27.3$ & $-27: 26: 49.9$ & +3.16 & -2.45 & 34.0 & 5084.92 & 62 & 5084.980 & 7200 & 20 & UVES & 45000 & TW \\
\hline MACHO-1999-BLG-022S & $18: 05: 05.8$ & $-28: 34: 39.5$ & +2.46 & -3.51 & 265.0 & 1365.50 & 28 & 1366.315 & 12600 & 80 & HIRES & 29000 & $\mathrm{C} 03$ \\
\hline OGLE-2008-BLG-209S & 18:04:50.0 & $-29: 42: 35.3$ & +1.44 & -4.01 & 19.5 & 4606.09 & 30 & 4606.833 & 5400 & 30 & MIKE & 47000 & $\mathrm{~B} 09 \mathrm{~b}$ \\
\hline MOA-2009-BLG-489S & $17: 57: 46.5$ & $-28: 38: 57.8$ & +1.61 & -2.14 & 58.9 & 5095.52 & 103 & 5094.982 & 7200 & 65 & UVES & 45000 & TW \\
\hline MOA-2009-BLG-456S & $17: 48: 56.3$ & $-34: 13: 32.3$ & -4.16 & -3.34 & 36.2 & 5090.94 & 77 & 5090.982 & 7200 & 45 & UVES & 45000 & TW \\
\hline OGLE-2007-BLG-514S & 17:58:03.0 & $-27: 31: 08.2$ & +2.62 & -1.63 & 76.0 & 4386.28 & 1200 & 4385.985 & 4800 & 30 & MIKE & 25000 & E10 \\
\hline MOA-2009-BLG-259S & $17: 57: 57.6$ & $-29: 11: 39.1$ & +1.15 & -2.45 & 69.1 & 5016.77 & 220 & 5016.227 & 7920 & 50 & UVES & 45000 & TW \\
\hline MOA-2008-BLG-311S & $17: 56: 53.7$ & $-31: 23: 40.3$ & -0.87 & -3.35 & 15.7 & 4655.40 & 400 & 4654.952 & 7200 & 85 & MIKE & 29000 & $\mathrm{C} 09$ \\
\hline MOA-2008-BLG-310S & $17: 54: 14.4$ & $-34: 46: 37.7$ & -4.09 & -4.56 & 7.1 & 4656.39 & 280 & 4655.957 & 7200 & 90 & MIKE & 41000 & $\mathrm{C} 09$ \\
\hline OGLE-2007-BLG-349S & $18: 05: 23.0$ & $-26: 25: 27.1$ & +4.38 & -2.52 & 109.4 & 4348.56 & 400 & 4348.237 & 3050 & 90 & HIRES & 48000 & $\mathrm{C} 08$ \\
\hline MOA-2006-BLG-099S & $17: 54: 10.2$ & $-35: 13: 34.9$ & -4.48 & -4.78 & 30.1 & 3940.35 & 380 & 3940.090 & 2400 & 30 & MIKE & 19000 & J08 \\
\hline OGLE-2006-BLG-265S & 18:07:18.9 & $-27: 47: 44.0$ & +3.38 & -3.55 & 28.6 & 3893.24 & 212 & 3892.581 & 900 & 45 & HIRES & 45000 & $\mathrm{~J} 07$ \\
\hline
\end{tabular}

Notes. ${ }^{(\dagger)}$ Given for each microlensing event is: RA and DE coordinates (J2000) read from the fits headers of the spectra (the direction where the telescope pointed during observation); galactic coordinates $(l$ and $b)$; duration of the event in days $\left(T_{\mathrm{E}}\right)$; time when maximum magnification occured $\left(T_{\max }\right)$; maximum magnification $\left(A_{\max }\right)$; time when event was observed with high-resolution spectrograph $\left(T_{\mathrm{obs}}\right)$; magnification at the time of observation $\left(A_{\mathrm{obs}}\right)$; the exposure time (Exp.), the measured signal-to-noise ration per pixel at $\sim 6400 \AA$; the spectrograph that was used; the spectral resolution; and the reference where the star first appeared: TW = This Work, B09a = Bensby et al. (2009a), B09b = Bensby et al. (2009b), J07 = Johnson et al. (2007), J08 = Johnson et al. (2008), C08 = Cohen et al. (2008), C09 = Cohen et al. (2009), C03 = Cavallo et al. (2003), E10 $=$ Epstein et al. (2010). ${ }^{(亠)}$ MACHO ID: 109.20893.3423.

CCD). In all cases a slit width of $1^{\prime \prime}$ was used, giving a resolving power of $R \approx 45000$.

On all occasions, right before or right after the observations of the microlensed targets in the Bulge, a rapidly rotating B star, either HR 6141 or HR 8431, was observed at an airmass similar to that of the Bulge stars. The featureless spectra from these B stars were used to divide out telluric lines in the spectra of the Bulge stars. Also, at the beginning of the night of April 11 we obtained a solar spectrum by observing the asteroid Pallas.

Data taken before the upgrade of the UVES red CCD in midJuly were reduced with the UVES pipeline (CPL version 3.9.0), while the data taken after the upgrade were reduced with version 4.4.5. Typical signal-to-noise ratios per pixel at $6400 \AA$ are given in Table 1.

The light curves for the seven microlensing events (including OGLE-2009-BLG-076S from Bensby et al. 2009a) observed with UVES in 2009 are shown in Fig. 1, in which we have also indicated the time interval during which they were observed with high-resolution spectrographs. Positions, amplifications, times of observation, and exposure times are given in Table 1.

\subsection{Los MACHOs}

Cavallo et al. (2003) presented the first detailed elemental abundance study of microlensed dwarf stars in the Bulge. Their analysis was of a "preliminary" nature, so we decided to re-analyse the stars that they classified as either dwarf or subgiant stars. There are four such stars: MACHO-1997-BLG045S, MACHO-1998-BLG-006S, MACHO-1999-BLG-001S, and MACHO-1999-BLG-022S.

The observations of these stars were carried out from 1997 to 1999 with the HIRES spectrograph on the Keck I telescope on Hawaii. By using a $1.148^{\prime \prime}$ wide slit and a $2 \times 2$ binning, spectra with a resolution of $R \approx 29000$ were obtained. These data were obtained when the HIRES detector had only a single CCD chip.
The data are now publicly available and we gathered science and associated calibration files from the Keck Observatory Archive ${ }^{4}$.

The data reduction was carried out using the LONG and ECHELLE contexts of the MIDAS ${ }^{5}$ software. Because the bias level is changing on a time scale of a few minutes, we used the over-scan region to compensate for the observed variations and to bring all the raw data to an effectively homogenised bias level. Master calibration frames were created by averaging the relevant frames obtained close in time to the science observations. The data were then bias, dark and background-illumination subtracted using standard procedures of the ECHELLE context. The orders were traced directly on the science images and a 5-pixel window was used to extract the object spectra. Sky spectra were extracted from two smaller windows on both sides of the object window. Flat-field and wavelength calibration spectra were extracted using the exact same windows as the one used for object and sky extraction. The science and sky spectra were then flatfielded and sky subtracted. Finally, because of little or no overlap between the orders, the wavelength calibration was performed individually for each order. In total, 27 orders were observed yielding an effective wavelength coverage from 4670 to $7180 \AA$, although with some gaps between the orders.

Only two of these four stars could be analysed. The reduced spectrum for MACHO-1997-BLG-045S was not of sufficient quality to allow for any measurements of equivalent widths or line synthesis necessary for a proper abundance analysis, and MACHO-1999-BLG-001S appears to be a spectroscopic binary. Also, as MACHO-1999-BLG-006S turned out to be a lowluminosity giant after our re-analysis $(\log g \approx 2-3$ ), the results for this star will be presented together with the other similar

\footnotetext{
4 Available at http://koa.ipac. caltech.edu

5 ESO-MIDAS is the acronym for the European Southern Observatory Munich Image Data Analysis System which is developed and maintained by the European Southern Observatory.
} 
T. Bensby et al.: Chemical evolution of the Galactic bulge as traced by microlensed dwarf and subgiant stars. II.

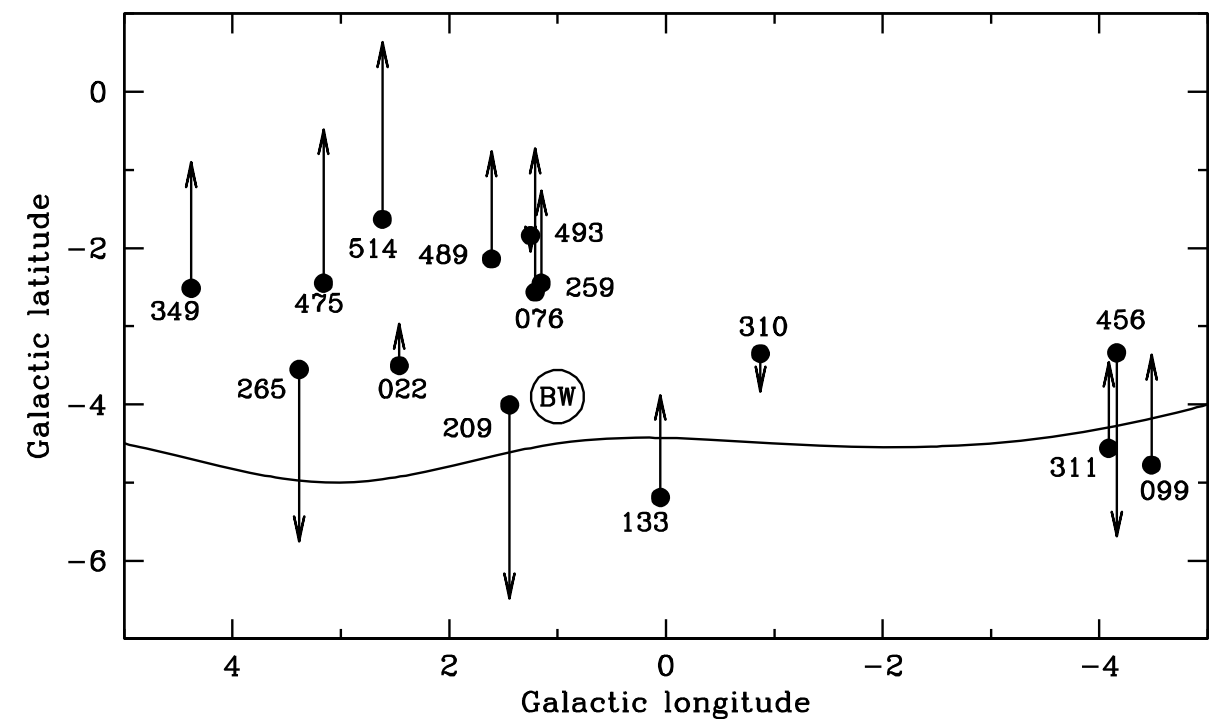

Fig. 2. Positions and radial velocities for the 15 microlensed stars. The arrows represent measured radial velocities and one degree corresponds to $70 \mathrm{~km} \mathrm{~s}^{-1}$. Upward pointing arrows indicate positive velocities. The curved line shows the outline of the southern Bulge based on observations with the COBE satellite (Weiland et al. 1994). The location of Baade's window $(\mathrm{BW})$ is marked by the larger open circle. Stars have been labelled with the last number in their IDs. low-luminosity giant stars observed at ESO in a subsequent study. The light curve for MACHO-1997-BLG-022S is shown in Fig. 1 and event data given in Table 1.

\section{Bulge membership}

\subsection{Positions on the sky and radial velocities}

The locations on the sky of the events are shown in Fig. 2. All the stars have negative Galactic latitudes because OGLE and MOA currently only monitor fields with $b<0$. The angular distances to the Galactic plane are similar for all events, between $2-5^{\circ}$. The measured radial velocities for the stars have been indicated in Fig. 2. Arrows upward means positive radial velocities, arrows downward negative velocities, and the scale in the figure is $70 \mathrm{~km} \mathrm{~s}^{-1}$ per degree. The high variation in $v_{\mathrm{r}}$ for the microlensed stars is consistent with the high velocity dispersion seen for Bulge giant stars (compare, e.g., the recent BRAVA radial velocity survey of red giants in the Bulge by Howard et al. 2008).

\subsection{Microlensing toward the Galactic bulge}

Because we need to observe the microlensing events wherever they occur in the central regions of the Galaxy, we cannot choose stars along, e.g., the minor axis to maximise the contribution of the Bulge, leading to possible confusion about whether these stars are Bulge, disc or halo stars.

Our current approach to this is to regard the division into Bulge and disc for stars within $1 \mathrm{kpc}$ of the Galactic centre as a semantic division. There is no evidence for a cold rotating extended disc that close to the Galactic centre for the fields that have been studied so far (Howard et al. 2009), and the creation of the Bulge from the Galactic disc is one of the scenarios we wish to test. So the question then becomes whether the microlensed dwarf and subgiant stars are located in the Bulge region, or in the disc on either this side or the far side of the Bulge. Nair \& Miralda-Escude (1999) estimate that about $15 \%$ of the events toward the Bulge could have source stars belonging to the far side of disc, more than $3 \mathrm{kpc}$ away from the Galactic centre. On the other hand, more recent theoretical calculations of the distance to microlensed sources, assuming a constant disc density and an exponential bulge, show that the distance to the sources is strongly peaked in the Bulge, with the probability of having $D<7$ kpc very small (Kane \& Sahu 2006).

Another argument that these are Bulge stars, rather than disc stars, are the large radial velocities for stars close to the Galactic centre (e.g. Epstein et al. 2010) as well as the fact that there are stars with radial velocities in opposite directions on the same side of the Galactic centre (e.g., Cohen et al. 2008).

In addition, we have prior knowledge of the colours and magnitudes of the source stars when unmagnified. The stars we observed were all identified as dwarf or subgiant stars at the distance of the Bulge based on OGLE or MOA instrumental colours and magnitudes, estimated from the offsets from the red clump stars in the same field. The parameters for the stars determined in this manner have been repeatedly tested against the parameters derived spectroscopically and the overall consistency between these results (e.g. Johnson et al. 2007, 2008) again shows that these stars are likely located at the distance of the Bulge and not the near or far disc. The fact that the latitudes of the stars are closer to the Galactic plane may make disc contamination more likely for unmagnified sources; however, the combination of kinematics, colour-magnitude diagrams and microlensing statistics indicate that we are studying a stellar population belonging in the Bulge.

\section{Analysis}

\subsection{Stellar parameters and elemental abundances}

The determination of stellar parameters and calculation of elemental abundances were carried out as described in method 1 of Bensby et al. (2009b). Briefly, this method is based on equivalent width measurements $\left(W_{\lambda}\right)$ and one-dimensional LTE model stellar atmospheres calculated with the Uppsala MARCS code (Gustafsson et al. 1975; Edvardsson et al. 1993; Asplund et al. 1997). The spectral line list is an expanded version of the list used by Bensby et al. $(2003,2005)$ and is in full given in Bensby et al. (in prep.). Equivalent widths were measured using the IRAF ${ }^{6}$ task SPLOT. Gaussian line profiles were fitted to the observed lines, but in special cases of strong $\mathrm{Mg}, \mathrm{Ca}, \mathrm{Si}$ 6 IRAF is distributed by the National Optical Astronomy Observatory,
which is operated by the Association of Universities for Research
in Astronomy, Inc., under co-operative agreement with the National Science Foundation. 

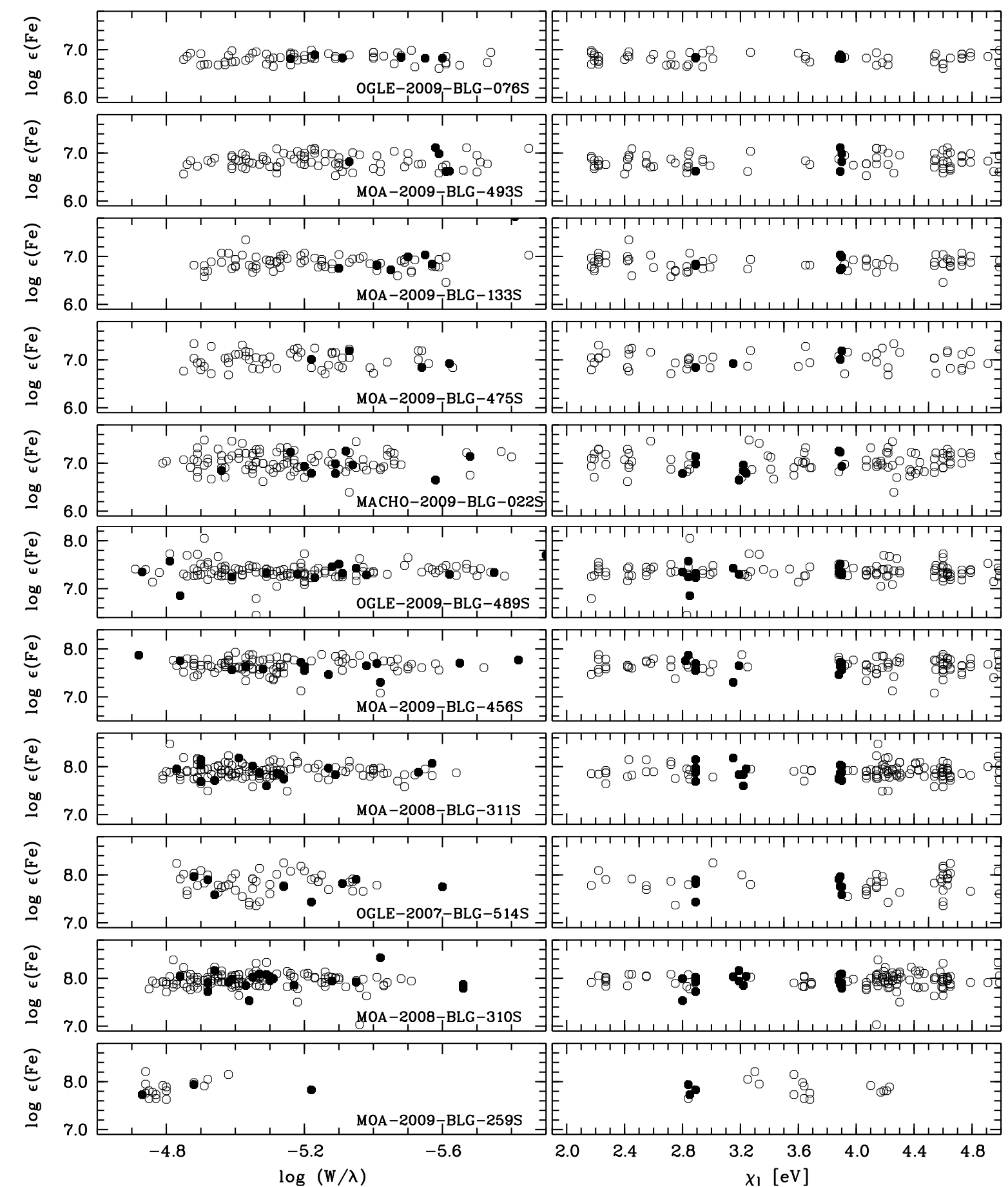

Fig. 3. Diagnostic plots showing absolute Fe abundances versus reduced line strength and lower excitation potential. Open circles indicate abundances from Fe I lines and filled circles from Fe II lines. Similar plots for MOA-2006-BLG-099S, OGLE-2006-BLG-265S, OGLE-2007-BLG349S, and OGLE-2008-BLG-209S can be found in Bensby et al. (2009b). Note the limited number of lines for MOA-2009-BLG-259S due to that this star was observed when only the UVES blue CCD was available.

and $\mathrm{Ba}$ lines, Voigt profiles were used to better account for the extended wing profiles of these lines.

The effective temperature $\left(T_{\text {eff }}\right)$ is found by requiring excitation balance of abundances from Fe I lines, the microturbulence parameter $\left(\xi_{\mathrm{t}}\right)$ by requiring zero slope in the graph where abundances from Fe I lines are plotted versus the reduced strength $\left(\log \left(W_{\lambda} / \lambda\right)\right)$ of the spectral lines, and the surface gravity $(\log g)$ from ionisation balance, i.e., requiring that the average abundances from Fe I and Fe II lines are equal. Only Fe I and
Fe II lines with measured equivalent widths smaller than $90 \mathrm{~m} \AA$ are used in the determination of the stellar parameters. Figure 3 shows the diagnostic plots, $\log \epsilon(\mathrm{Fe})$ versus $\log \left(W_{\lambda} / \lambda\right)$ and lower excitation potential $\left(\chi_{\mathrm{e}}\right)$, for the stars.

To relate the elemental abundances to those in the Sun we determine our own solar abundances. The equivalent widths we measure in the solar spectrum that was obtained by observing the asteroid Pallas with UVES on April 11, 2009, show very good agreement with the equivalent widths of several solar 
T. Bensby et al.: Chemical evolution of the Galactic bulge as traced by microlensed dwarf and subgiant stars. II.

Table 2. Stellar parameters, ages, and radial velocities for the sample of microlensed dwarf stars ${ }^{\dagger}$.

\begin{tabular}{rlcccrrrr}
\hline \hline Object & \multicolumn{1}{c}{$\begin{array}{c}T_{\mathrm{eff}} \\
{[\mathrm{K}]}\end{array}$} & $\begin{array}{c}\log g \\
{[\mathrm{cgs}]}\end{array}$ & $\begin{array}{c}\xi_{\mathrm{t}} \\
{\left[\mathrm{km} \mathrm{s}^{-1}\right]}\end{array}$ & {$[\mathrm{Fe} / \mathrm{H}]$} & $N_{\mathrm{Fe} I}, N_{\mathrm{Fe} \text { II }}$ & $\begin{array}{c}\text { Age } \\
{[\mathrm{Gyr}]}\end{array}$ & $\begin{array}{c}v_{\mathrm{r}} \\
{\left[\mathrm{km} \mathrm{s}{ }^{-1}\right]}\end{array}$ \\
\hline OGLE-2009-BLG-076S & $5877 \pm 96$ & $4.30 \pm 0.18$ & $1.61 \pm 0.16$ & $-0.72 \pm 0.07$ & 66, & 7 & $11.7 \pm 1.9$ & +128.7 \\
MOA-2009-BLG-493S & $5457 \pm 98$ & $4.50 \pm 0.22$ & $0.83 \pm 0.30$ & $-0.70 \pm 0.14$ & 80, & 5 & $9.1 \pm 4.0$ & -14.5 \\
MOA-2009-BLG-133S & $5597 \pm 92$ & $4.40 \pm 0.27$ & $1.15 \pm 0.27$ & $-0.64 \pm 0.17$ & 68, & 7 & $9.4 \pm 4.0$ & +91.6 \\
MOA-2009-BLG-475S & $5843 \pm 173$ & $4.40 \pm 0.30$ & $1.31 \pm 0.31$ & $-0.54 \pm 0.17$ & 53, & 4 & $9.1 \pm 3.7$ & +137.8 \\
MACHO-1999-BLG-022S & $5650 \pm 106$ & $4.05 \pm 0.20$ & $0.30 \pm 0.45$ & $-0.49 \pm 0.14$ & 97, & 10 & $11.7 \pm 2.3$ & +37.6 \\
OGLE-2008-BLG-209S & $5243 \pm 65$ & $3.82 \pm 0.16$ & $1.01 \pm 0.11$ & $-0.30 \pm 0.06$ & 146, & 19 & $9.5 \pm 3.1$ & -173.6 \\
MOA-2009-BLG-489S & $5634 \pm 89$ & $4.30 \pm 0.18$ & $0.68 \pm 0.17$ & $-0.18 \pm 0.11$ & 114, & 15 & $11.0 \pm 2.3$ & +96.5 \\
MOA-2009-BLG-456S & $5700 \pm 93$ & $4.24 \pm 0.18$ & $1.00 \pm 0.20$ & $+0.12 \pm 0.09$ & 91, & 14 & $7.9 \pm 1.6$ & -164.6 \\
OGLE-2007-BLG-514S & $5644 \pm 130$ & $4.10 \pm 0.28$ & $1.55 \pm 0.29$ & $+0.27 \pm 0.09$ & 49, & 8 & $6.9 \pm 1.6$ & +158.8 \\
MOA-2009-BLG-259S & $5000 \pm 400$ & $4.50 \pm 0.50$ & $0.50 \pm 0.75$ & $+0.33 \pm 0.40$ & 16, & 3 & $9.4 \pm 4.0$ & +83.2 \\
MOA-2008-BLG-311S & $5944 \pm 68$ & $4.40 \pm 0.13$ & $1.17 \pm 0.12$ & $+0.36 \pm 0.07$ & 118, & 16 & $2.3 \pm 1.0$ & -34.1 \\
MOA-2008-BLG-310S & $5704 \pm 65$ & $4.30 \pm 0.12$ & $1.05 \pm 0.11$ & $+0.42 \pm 0.08$ & 122, & 20 & $4.5 \pm 1.0$ & +77.5 \\
OGLE-2007-BLG-349S & $5229 \pm 63$ & $4.18 \pm 0.13$ & $0.78 \pm 0.13$ & $+0.42 \pm 0.08$ & 103, & 18 & $13.6 \pm 1.0$ & +113.0 \\
MOA-2006-BLG-099S & $5741 \pm 87$ & $4.47 \pm 0.15$ & $0.84 \pm 0.14$ & $+0.44 \pm 0.10$ & 119, & 21 & $2.8 \pm 1.3$ & +99.0 \\
OGLE-2006-BLG-265S & $5486 \pm 70$ & $4.24 \pm 0.15$ & $1.17 \pm 0.12$ & $+0.47 \pm 0.06$ & 92, & 14 & $7.9 \pm 1.3$ & -154.0 \\
\hline
\end{tabular}

Notes. ${ }^{(\dagger)}$ For the four stars analysed in Bensby et al. (2009b): OGLE-2008-BLG-209S, MOA-2006-BLG-099S, OGLE-2006-BLG-265S, and OGLE-2007-BLG-349S, we have here updated the estimations of the uncertainties using the method outlined in Epstein et al. (2010).

spectra (average of Ganymede, Ceres, Vesta, Moon, and sky spectra) in Bensby et al. (in prep.). On average the measurements of the Pallas solar spectrum is only $0.3 \%$ larger, which is truly negligible. Hence, to ensure that the normalised abundances for the microlensed dwarf stars are on the same baseline as the sample of $\sim 700$ thin and thick disc dwarf stars in Bensby et al. (in prep.) we use the average equivalent widths based on measurements in all solar spectra (see Bensby et al. in prep.).

Final abundances are normalised on a line-by-line basis and then we take the median value for each element. In a few cases when the equivalent width of an Fe line in the Sun was larger than $90 \mathrm{~m} \AA$, or when a Ti or Cr line were larger than $110 \mathrm{~m} \AA$, and these lines were measured in the Bulge dwarf star, we normalised the abundance for that line with the average abundance from all other lines that were measured in the solar spectrum for that element. These cases are marked by "av" in Col. 7 in Table 4.

Final stellar parameters for our targets are given in Table 2. All measured equivalent widths and elemental abundances for individual spectral lines are given in Table 4, while Table 5 gives the normalised abundance ratios.

\subsection{Additional dwarf stars}

In order to increase the sample size of microlensed dwarf stars, we include the two stars MOA-2009-BLG-310S, and -311S, recently published by Cohen et al. (2009), and OGLE-2007-BLG514 S by Epstein et al. (2010). The metallicities that were found for these three stars by Cohen et al. (2009) and Epstein et al. (2010) are $[\mathrm{Fe} / \mathrm{H}]=+0.41,[\mathrm{Fe} / \mathrm{H}]=+0.26$, and $[\mathrm{Fe} / \mathrm{H}]=$ +0.33 , respectively.

The spectra for these stars were kindly provided by the authors and we have re-analysed them using our methods in order to have all 15 microlensed dwarf stars on the same baseline. The values we find for these stars are listed in Table 2, and they are generally in good agreement with what were found in Cohen et al. (2009) and Epstein et al. (2010). The main differences are that we derive a $240 \mathrm{~K}$ higher $T_{\text {eff }}$ and 0.2 dex higher $\log g$ for MOA-2009-BLG-311S, and a 0.2 dex lower $\log g$ for OGLE2007-BLG-514S. The other differences are within the estimated uncertainties.

\subsection{Error analysis}

A rigorous error analysis as outlined in Epstein et al. (2010) has been performed for the microlensed dwarf stars. This method takes into account the uncertainties in the four observables that were used to find the stellar parameters, i.e. the uncertainty of the slope in the graph of Fe I abundances versus lower excitation potential; the uncertainty of the slope in the graph of Fe I abundances versus line strength; the uncertainty in the difference between Fe I and Fe II abundances; and the uncertainty in the difference between input and output metallicities. The method also accounts for abundance spreads (line-to-line scatter) as well as how the abundances for each element reacts to changes in the stellar parameters.

The resulting errors in the stellar parameters are given together with the best fit values of the stellar parameters in Table 2. The errors in the abundance ratios are given in Table 5.

\subsection{Stellar ages}

Stellar ages were determined as described in Meléndez et al. (2009). We interpolated a fine grid of $\alpha$-enhanced Yonsei-Yale $\left(\mathrm{Y}^{2}\right)$ isochrones by Demarque et al. (2004), adopting $[\alpha / \mathrm{Fe}]=0$ for $[\mathrm{Fe} / \mathrm{H}]>0,[\alpha / \mathrm{Fe}]=-0.3 \times[\mathrm{Fe} / \mathrm{H}]$ for $-1 \leq[\mathrm{Fe} / \mathrm{H}] \leq 0$, and $[\alpha / \mathrm{Fe}]=+0.3$ for $[\mathrm{Fe} / \mathrm{H}]<-1$. At a given metallicity, we searched for all solutions allowed by the error bars in $T_{\text {eff }}, \log g$ and $[\mathrm{Fe} / \mathrm{H}]$, adopting as final result the median age and as error the standard deviation. Column 6 in Table 2 give the median age, and Col. 7 the error. Figure 4 shows the 15 microlensed dwarf stars together with the $\mathrm{Y}^{2}$ isochrones in the $\log T_{\mathrm{eff}}-\log g$ plane.

\subsection{Check 1: temperatures from microlensing techniques}

De-reddened colours and magnitudes of the sources can be estimated using standard microlensing techniques (e.g. Yoo et al. 2004). The method for determining the colour does not make any assumption about the absolute reddening, nor about the ratio of selective to total extinction. It only assumes that the reddening toward the microlensed source is the same as the reddening toward the red clump, and that the red clump in the 
Table 3. Comparison of colours and effective temperatures as determined from spectroscopy and microlensing techniques ${ }^{\dagger}$.

\begin{tabular}{cccccc}
\hline \hline Object & $M_{I}$ & $(V-I)_{0}$ & $T_{\text {eff }}^{\text {phot }}$ & $T_{\text {eff }}$ & $(V-I)_{0}^{\text {spec }}$ \\
\hline OGLE-2009-BLG-076S & 4.19 & 0.67 & 5775 & 5877 & 0.65 \\
MOA-2009-BLG-493S & 3.34 & 0.79 & 5325 & 5457 & 0.75 \\
MOA-2009-BLG-133S & 4.19 & 0.68 & 5730 & 5597 & 0.71 \\
MOA-2009-BLG-475S & 4.25 & 0.59 & 6150 & 5843 & 0.65 \\
MACHO-1999-BLG-022S & - & - & - & 5650 & 0.71 \\
OGLE-2008-BLG-209S & 2.52 & 0.71 & 5670 & 5243 & 0.82 \\
MOA-2009-BLG-489S & 3.39 & 0.85 & 5200 & 5634 & 0.71 \\
MOA-2009-BLG-456S & 2.76 & 0.66 & 5870 & 5700 & 0.71 \\
OGLE-2007-BLG-514S & 4.68 & 0.70 & 5760 & 5644 & 0.73 \\
MOA-2009-BLG-259S & 2.91 & 0.79 & 5450 & 5000 & 0.97 \\
MOA-2008-BLG-311S & 3.85 & 0.66 & 5880 & 5944 & 0.65 \\
MOA-2008-BLG-310S & 3.46 & 0.69 & 5780 & 5704 & 0.72 \\
OGLE-2007-BLG-349S & 4.54 & 0.78 & 5490 & 5229 & 0.87 \\
MOA-2006-BLG-099S & 3.81 & 0.74 & 5620 & 5741 & 0.70 \\
OGLE-2006-BLG-265S & 3.59 & 0.68 & 5840 & 5486 & 0.78 \\
\hline
\end{tabular}

Notes. ${ }^{(\dagger)}$ Columns 2 and 3 give the absolute dereddened magnitudes and colours based on microlensing techniques; based on the colours in Col. 3; Col. 4 gives the inferred effective temperatures using the colour-[Fe/H] $-T_{\text {eff }}$ calibrations by Ramírez \& Meléndez (2005); Col. 5 gives our spectroscopic temperatures (same as in Col. 2 in Table 2); and Col. 6 gives the colours, based on the colour- $[\mathrm{Fe} / \mathrm{H}]-T_{\text {eff }}$ calibrations by Ramírez \& Meléndez (2005), that the spectroscopic temperatures in Col. 5 gives.

Bulge has $(V-I)_{0}=1.05$ and $I_{0}=14.32$ (e.g., Johnson et al. 2008; Epstein et al. 2010). The absolute de-reddened magnitude and colour are then derived from the offsets between the microlensing source and the red clump in the instrumental colour-magnitude diagram (CMD). The absolute de-reddened magnitudes and colours for 14 of the 15 microlensed stars are given in Table 3. Photometry for MACHO-1999-BLG-022S could not be recovered at this time.

From the colour- $[\mathrm{Fe} / \mathrm{H}]-T_{\text {eff }}$ calibrations by Ramírez \& Meléndez (2005) we check what temperature we should expect given the de-reddened colour and the metallicity we determined. On average we find that the spectroscopic temperatures are $103 \mathrm{~K}$ lower than the ones based on the colour- $[\mathrm{Fe} / \mathrm{H}]-T_{\text {eff }}$ relationships. The top panel of Fig. 5 shows a comparison between the two as a function of $[\mathrm{Fe} / \mathrm{H}]$. No obvious trends can be seen.

It is also possible to use the the same calibrations by Ramírez \& Meléndez (2005) to see what $(V-I)$ colours the spectroscopic effective temperatures and metallicities would give. These are listed in the last column of Table 3 , and the comparison between photometric and "spectroscopic" $(V-I)$ colours are shown in the bottom panel of Fig. 5. On average the spectroscopic colours are 0.03 mag higher, with no discernible trend with metallicity.

The offset that we see between spectroscopic and photometric values could be a result of the assumed magnitudes and colours of the red clump in the Bulge. Previously, it was assumed that the red clump stars in the Bulge had the same colour as the red clump stars in the Solar neighbourhood $\left((V-I)_{0}=1.00\right)$. Based on the first microlensing events of dwarf stars in the Bulge (Johnson et al. 2008; Cohen et al. 2008), and additional observational evidence (Epstein et al. 2010), this value was revised to $(V-I)_{0}=1.05$. Assuming that the spectroscopic temperatures are the correct ones, our results indicate that the $(V-I)_{0}$ colour of the red clump stars in the Bulge should be revised upwards by an additional few hundredths of a dex to $(V-I)_{0}=1.08$.

\subsection{Check 2: trends with metallicity}

Figure 6 shows how $T_{\text {eff }}$ and $\log g$ vary with metallicity. Regression lines, taking the errors in both $x$ and $y$ directions into account, are shown, as well as the regression parameters and their uncertainties. No significant trends with metallicity can be seen.

\subsection{Check 3: signal-to-noise and continuum bias}

The two papers by Bensby et al. (2009a,b) found the two first microlensed dwarf stars with sub-solar iron abundances. In this study we find an additional 5 stars with sub-solar $[\mathrm{Fe} / \mathrm{H}]$. It has been suggested that the reason for this could be that these spectra have, on average, lower $S / N$ than the other events studied, especially compared to Cohen et al. (2009) which all have very high $[\mathrm{Fe} / \mathrm{H}]$. The lower $S / N$ should then result in that the continuum was set too low and thus the $W_{\lambda}$ s underestimated, resulting in too low $[\mathrm{Fe} / \mathrm{H}]$.

We looked in greater detail at MOA-2009-BLG-475S, the dwarf star with the spectrum that has the lowest $S / N$, and tested if we could make the star more metal-rich. This experiment was done by assuming that the star is actually metal-rich and that there are many weak lines that makes it difficult to identify the level of the continuum. The best way to set the continuum is then to assume that the high points in the spectrum are indeed the continuum. We re-measured the star under this assumption and determined new stellar parameters. On average the equivalent widths became $10 \mathrm{~m} \AA$ larger. This resulted in a change of the metallicity of +0.1 dex, but the other stellar parameters $(\log g$, $T_{\text {eff }}$, and $\xi_{\mathrm{t}}$ ) were intact and did not change. Hence, we find it unlikely that our low-metallicity stars could be high-metallicity stars resulting from an erroneous analysis of their relatively low $S / N$ spectra.

\subsection{In summary}

Our analysis shows that the eight microlensed stars that we observed were successfully selected to be dwarf stars, varying in metallicity from $[\mathrm{Fe} / \mathrm{H}]=-0.72$ to +0.54 . The first results for OGLE-2009-BLG-076S were presented in Bensby et al. (2009a) where we found a metallicity of $[\mathrm{Fe} / \mathrm{H}]=-0.76$. The refined analysis in this study gives a metallicity of $[\mathrm{Fe} / \mathrm{H}]=-0.72$, and it still holds (barely) the place as the most metal-poor dwarf star in the Bulge, with MOA-2009-BLG-133S just 0.01 dex higher.

The uncertainties in the stellar parameters are below or around $100 \mathrm{~K}$ in $T_{\text {eff }}$, around $0.2 \mathrm{dex}$ in $\log g$, and around $0.1-0.2$ dex in $[\mathrm{Fe} / \mathrm{H}]$ (see Table 2). The clear exception is MOA-2009-BLG-259S where errors are exceptionally large. This is due to difficulties arising from the very limited wavelength coverage of the UVES spectrum that was obtained when only the blue CCD was available. The fact that it also turned out to be a metal-rich star at $[\mathrm{Fe} / \mathrm{H}]=+0.32$, further increases the errors due to line blending and uncertainties in the placement of the continuum. Also, the lack of weak lines, due to the high $[\mathrm{Fe} / \mathrm{H}]$, made it especially difficult to determine the microturbulence parameter (see Fig. 3). A spectrum covering the whole optical region of MOA-2009-BLG-259S was obtained by another group using the HIRES spectrograph, and they find a $0.2 \mathrm{dex}$ higher metallicity than what we do (Cohen et al. 2010). Although the metallicity is in reasonable agreement with what others have found we think that the errors in $T_{\text {eff }}$ and $\log g$ are so large that this star is not conveying any information in the $[\mathrm{Fe} / \mathrm{H}]$, age, or abundance plots. Therefore we do not include this star in the following discussions. 
T. Bensby et al.: Chemical evolution of the Galactic bulge as traced by microlensed dwarf and subgiant stars. II.

Table 4. Measured equivalent widths and calculated elemental abundances for each star ${ }^{\dagger}$.

\begin{tabular}{|c|c|c|c|c|c|c|c|c|c|c|c|c|c|c|c|c|c|c|c|c|c|}
\hline \multirow[b]{2}{*}{ El. } & \multirow[b]{2}{*}{$\lambda$} & \multirow[b]{2}{*}{$\chi_{1}$} & \multicolumn{3}{|c|}{ Sun } & \multicolumn{2}{|c|}{ ob09076 } & \multicolumn{2}{|c|}{ mb09493 } & \multicolumn{2}{|c|}{ mb09133 } & \multicolumn{2}{|c|}{ mb09475 } & \multicolumn{2}{|c|}{ mb99022 } & \multicolumn{2}{|c|}{ mb09489 } & \multicolumn{2}{|c|}{ mb09456 } & \multicolumn{2}{|c|}{ mb09259 } \\
\hline & & & $W_{\lambda}$ & $X$ & flg & $W_{\lambda}$ & $X$ & $W_{\lambda}$ & $X$ & $W_{\lambda}$ & $X$ & $W_{\lambda}$ & $X$ & $W_{\lambda}$ & $X$ & $W_{\lambda}$ & $X$ & $W_{\lambda}$ & $X$ & $W_{\lambda}$ & $X$ \\
\hline Al I & 6696.023 & 3.14 & 38.3 & 6.51 & 0 & - & - & 20.6 & 5.99 & 20.4 & 6.06 & - & - & 28.6 & 6.30 & 37.5 & 6.45 & 47.7 & 6.64 & - & - \\
\hline $\mathrm{Al} \mathrm{I}$ & 6698.673 & 3.14 & 20.7 & 6.47 & 0 & - & - & 12.3 & 6.04 & 7.5 & 5.88 & - & - & 17.3 & 6.32 & 20.3 & 6.39 & 33.6 & 6.70 & - & - \\
\hline$\vdots$ & : & $\vdots$ & & : & $\vdots$ & i & $\vdots$ & & : & & & : & $\vdots$ & & & & $\vdots$ & & : & & \\
\hline
\end{tabular}

Notes. ${ }^{(\dagger)}$ For each spectral line we give the lower excitation potential $\left(\chi_{1}\right)$, measured equivalent widths $\left(W_{\lambda}\right)$, derived absolute abundance $(X \equiv$ $\log \epsilon(X))$. We have only included the stars that are new to this work. The table is only available in electronic form at the CDS.
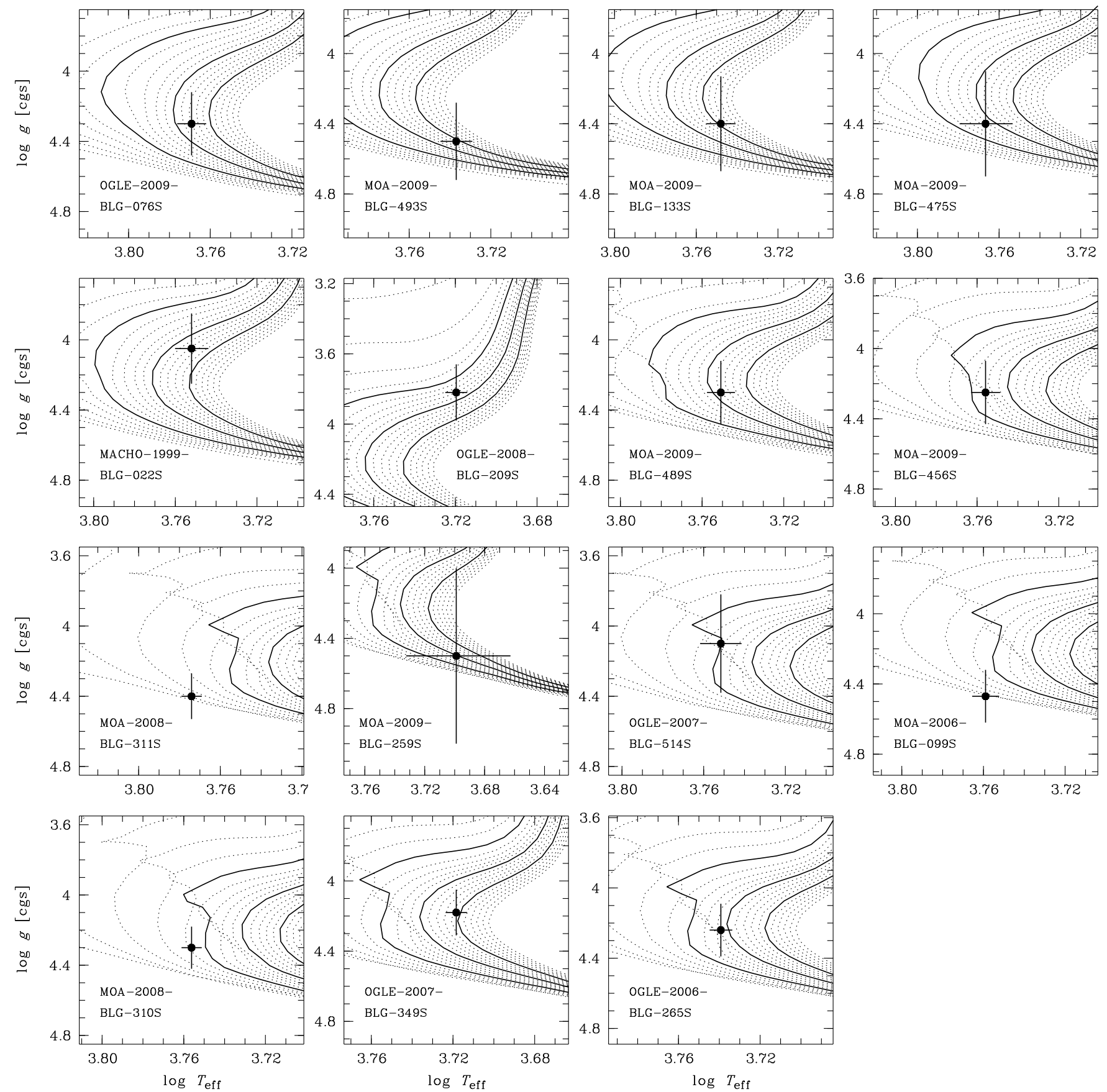

Fig. 4. Illustration of the estimation of stellar ages using the $\alpha$-enhanced isochrones from Demarque et al. (2004). Each set of isochrones have been calculated with the same metallicity and $\alpha$-enhancement as derived for the stars. In each plot the solid lines represent isochrones with ages of 5, 10, and $15 \mathrm{Gyr}$ (from left to right). Dotted lines are isochrones in steps of $1 \mathrm{Gyr}$, ranging from $0.1 \mathrm{Gyr}$ to 20 Gyr. Error bars represent the uncertainties in $T_{\text {eff }}$ and $\log g$ as given in Table 2. 
Table 5. Elemental abundance ratios, errors in the abundance ratios, and number of lines used, for 13 of the 15 microlensed dwarf stars ${ }^{\dagger}$.

\begin{tabular}{|c|c|c|c|c|c|c|c|c|c|c|c|c|c|}
\hline & {$[\mathrm{Fe} / \mathrm{H}]$} & {$[\mathrm{O} / \mathrm{Fe}]^{\ddagger}$} & {$[\mathrm{Na} / \mathrm{Fe}]$} & {$[\mathrm{Mg} / \mathrm{Fe}]$} & {$[\mathrm{Al} / \mathrm{Fe}]$} & {$[\mathrm{Si} / \mathrm{Fe}]$} & {$[\mathrm{Ca} / \mathrm{Fe}]$} & {$[\mathrm{Ti} / \mathrm{Fe}]$} & {$[\mathrm{Cr} / \mathrm{Fe}]$} & {$[\mathrm{Ni} / \mathrm{Fe}]$} & {$[\mathrm{Zn} / \mathrm{Fe}]$} & {$[\mathrm{Y} / \mathrm{Fe}]$} & {$[\mathrm{Ba} / \mathrm{Fe}]$} \\
\hline OGLE-2009-BLG-076S & -0.72 & 0.50 & 0.11 & 0.36 & 0.30 & 0.24 & 0.34 & 0.30 & - & 0.07 & - & - & -0.07 \\
\hline MOA-2009-BLG-493S & -0.70 & 0.43 & 0.13 & 0.37 & 0.28 & 0.18 & 0.17 & 0.34 & 0.01 & 0.04 & 0.25 & - & -0.07 \\
\hline MOA-2009-BLG-133S & -0.64 & 0.47 & 0.13 & 0.39 & 0.28 & 0.19 & 0.21 & 0.26 & -0.01 & 0.06 & - & - & -0.19 \\
\hline MOA-2009-BLG-475S & -0.52 & 0.33 & - & 0.25 & 0.23 & 0.18 & 0.14 & 0.33 & - & 0.07 & - & - & -0.18 \\
\hline MACHO-1999-BLG-022S & -0.49 & - & 0.01 & 0.35 & 0.31 & 0.25 & 0.18 & 0.25 & -0.03 & 0.00 & - & 0.01 & 0.15 \\
\hline OGLE-2008-BLG-209S & -0.30 & 0.34 & 0.08 & 0.34 & 0.24 & 0.20 & 0.18 & 0.29 & 0.05 & 0.05 & 0.16 & -0.08 & 0.05 \\
\hline MOA-2009-BLG-489S & -0.18 & 0.18 & -0.01 & 0.24 & 0.12 & 0.07 & 0.09 & 0.16 & 0.04 & 0.01 & 0.17 & -0.05 & -0.07 \\
\hline MOA-2009-BLG-456S & 0.12 & -0.12 & -0.03 & 0.09 & 0.08 & -0.01 & 0.00 & 0.08 & -0.06 & -0.03 & -0.03 & - & -0.08 \\
\hline MOA-2008-BLG-311S & 0.36 & -0.27 & 0.12 & 0.03 & 0.08 & 0.05 & 0.00 & 0.04 & -0.01 & 0.12 & 0.06 & 0.11 & -0.15 \\
\hline MOA-2008-BLG-310S & 0.42 & -0.27 & 0.08 & 0.08 & 0.10 & 0.02 & -0.03 & 0.09 & 0.05 & 0.06 & -0.03 & 0.06 & 0.03 \\
\hline OGLE-2007-BLG-349S & 0.42 & -0.14 & 0.15 & 0.05 & 0.09 & 0.12 & -0.06 & 0.02 & -0.03 & 0.11 & 0.11 & -0.12 & -0.13 \\
\hline MOA-2008-BLG-099S & 0.44 & -0.10 & 0.05 & 0.04 & -0.02 & 0.00 & -0.05 & -0.01 & -0.16 & -0.02 & 0.05 & -0.04 & 0.00 \\
\hline \multirow[t]{2}{*}{ OGLE-2006-BLG-265S } & 0.47 & -0.25 & 0.14 & 0.13 & 0.08 & 0.06 & -0.05 & -0.06 & -0.07 & 0.07 & -0.05 & 0.02 & -0.08 \\
\hline & $\sigma_{[\mathrm{Fe} / \mathrm{H}]}$ & $\sigma_{[\mathrm{O} / \mathrm{Fe}]}$ & $\sigma_{[\mathrm{Na} / \mathrm{Fe}]}$ & $\sigma_{[\mathrm{Mg} / \mathrm{Fe}]}$ & $\sigma_{[\mathrm{Al} / \mathrm{Fe}]}$ & $\sigma_{[\mathrm{Si} / \mathrm{Fe}]}$ & $\sigma_{[\mathrm{Ca} / \mathrm{Fe}]}$ & $\sigma_{[\mathrm{Ti} / \mathrm{Fe}]}$ & $\sigma_{[\mathrm{Cr} / \mathrm{Fe}]}$ & $\sigma_{[\mathrm{Ni} / \mathrm{Fe}]}$ & $\sigma_{[\mathrm{Zn} / \mathrm{Fe}]}$ & $\sigma_{[\mathrm{Y} / \mathrm{Fe}]}$ & $\sigma_{[\mathrm{Ba} / \mathrm{Fe}]}$ \\
\hline OGLE-2009-BLG-076S & 0.07 & 0.17 & 0.04 & 0.06 & 0.05 & 0.06 & 0.05 & 0.03 & - & 0.09 & - & - & 0.10 \\
\hline MOA-2009-BLG-493S & 0.14 & 0.51 & 0.07 & 0.10 & 0.08 & 0.19 & 0.30 & 0.08 & 0.10 & 0.17 & 0.38 & - & 0.18 \\
\hline MOA-2009-BLG-133S & 0.17 & 0.62 & 0.13 & 0.21 & 0.07 & 0.12 & 0.36 & 0.06 & 0.16 & 0.15 & - & - & 0.51 \\
\hline MOA-2009-BLG-475S & 0.17 & 0.39 & - & 0.09 & 0.09 & 0.10 & 0.18 & 0.08 & - & 0.15 & - & - & 0.31 \\
\hline MACHO-1999-BLG-022S & 0.14 & - & 0.07 & 0.13 & 0.10 & 0.13 & 0.13 & 0.24 & 0.14 & 0.10 & 0.19 & 0.31 & 0.12 \\
\hline OGLE-2008-BLG-209S & 0.06 & 0.22 & 0.07 & 0.10 & 0.04 & 0.08 & 0.12 & 0.08 & 0.09 & 0.07 & 0.18 & 0.20 & 0.08 \\
\hline MOA-2009-BLG-489S & 0.11 & 0.25 & 0.12 & 0.07 & 0.05 & 0.09 & 0.12 & 0.08 & 0.06 & 0.08 & 0.09 & 0.34 & 0.10 \\
\hline MOA-2009-BLG-456S & 0.09 & 0.21 & 0.05 & 0.08 & 0.05 & 0.07 & 0.10 & 0.05 & 0.09 & 0.08 & 0.17 & - & 0.09 \\
\hline MOA-2008-BLG-311S & 0.07 & 0.18 & 0.12 & 0.09 & 0.05 & 0.04 & 0.11 & 0.10 & 0.07 & 0.05 & 0.13 & 0.19 & 0.11 \\
\hline MOA-2008-BLG-310S & 0.08 & 0.16 & 0.10 & 0.09 & 0.07 & 0.05 & 0.10 & 0.08 & 0.06 & 0.05 & 0.13 & 0.23 & 0.09 \\
\hline OGLE-2007-BLG-349S & 0.08 & 0.19 & 0.15 & 0.14 & 0.08 & 0.07 & 0.12 & 0.07 & 0.05 & 0.04 & 0.10 & 0.17 & 0.08 \\
\hline MOA-2006-BLG-099S & 0.10 & 0.20 & 0.11 & 0.10 & 0.04 & 0.07 & 0.11 & 0.11 & 0.08 & 0.06 & 0.10 & 0.30 & 0.10 \\
\hline \multirow[t]{2}{*}{ OGLE-2006-BLG-265S } & 0.06 & 0.17 & 0.11 & 0.10 & 0.05 & 0.06 & 0.11 & 0.07 & 0.06 & 0.06 & 0.43 & 0.23 & 0.08 \\
\hline & $\sigma_{[\mathrm{Fe} / \mathrm{H}]}$ & $\sigma_{[\mathrm{O} / \mathrm{H}]}$ & $\sigma_{[\mathrm{Na} / \mathrm{H}]}$ & $\sigma_{[\mathrm{Mg} / \mathrm{H}]}$ & $\sigma_{[\mathrm{Al} / \mathrm{H}]}$ & $\sigma_{[\mathrm{Si} / \mathrm{H}]}$ & $\sigma_{[\mathrm{Ca} / \mathrm{H}]}$ & $\sigma_{[\mathrm{Ti} / \mathrm{H}]}$ & $\sigma_{[\mathrm{Cr} / \mathrm{H}]}$ & $\sigma_{[\mathrm{Ni} / \mathrm{H}]}$ & $\sigma_{[\mathrm{Zn} / \mathrm{H}]}$ & $\sigma_{[\mathrm{Y} / \mathrm{H}]}$ & $\sigma_{[\mathrm{Ba} / \mathrm{H}]}$ \\
\hline OGLE-2009-BLG-076S & 0.07 & 0.10 & 0.05 & 0.06 & 0.05 & 0.05 & 0.10 & 0.08 & - & 0.06 & - & - & 0.11 \\
\hline MOA-2009-BLG-493S & 0.14 & 0.38 & 0.09 & 0.16 & 0.07 & 0.06 & 0.43 & 0.13 & 0.07 & 0.05 & 0.26 & - & 0.18 \\
\hline MOA-2009-BLG-133S & 0.17 & 0.46 & 0.05 & 0.36 & 0.18 & 0.06 & 0.52 & 0.12 & 0.07 & 0.04 & - & - & 0.36 \\
\hline MOA-2009-BLG-475S & 0.17 & 0.23 & - & 0.17 & 0.13 & 0.08 & 0.31 & 0.14 & - & 0.07 & - & - & 0.21 \\
\hline MACHO-1999-BLG-022S & 0.14 & _ & 0.14 & 0.23 & 0.06 & 0.06 & 0.25 & 0.16 & 0.11 & 0.08 & 0.10 & 0.22 & 0.15 \\
\hline OGLE-2008-BLG-209S & 0.06 & 0.16 & 0.11 & 0.15 & 0.07 & 0.03 & 0.17 & 0.06 & 0.06 & 0.03 & 0.16 & 0.16 & 0.08 \\
\hline MOA-2009-BLG-489S & 0.11 & 0.15 & 0.13 & 0.14 & 0.07 & 0.03 & 0.21 & 0.09 & 0.07 & 0.04 & 0.06 & 0.29 & 0.09 \\
\hline MOA-2009-BLG-456S & 0.09 & 0.14 & 0.07 & 0.12 & 0.09 & 0.04 & 0.15 & 0.10 & 0.14 & 0.04 & 0.15 & - & 0.10 \\
\hline MOA-2008-BLG-311S & 0.07 & 0.12 & 0.17 & 0.14 & 0.10 & 0.06 & 0.17 & 0.07 & 0.06 & 0.04 & 0.07 & 0.13 & 0.07 \\
\hline MOA-2008-BLG-310S & 0.08 & 0.10 & 0.14 & 0.13 & 0.09 & 0.04 & 0.15 & 0.08 & 0.05 & 0.05 & 0.08 & 0.21 & 0.12 \\
\hline OGLE-2007-BLG-349S & 0.08 & 0.13 & 0.19 & 0.18 & 0.08 & 0.04 & 0.17 & 0.09 & 0.07 & 0.06 & 0.10 & 0.17 & 0.12 \\
\hline MOA-2006-BLG-099S & 0.10 & 0.11 & 0.18 & 0.18 & 0.08 & 0.05 & 0.20 & 0.07 & 0.06 & 0.07 & 0.09 & 0.25 & 0.09 \\
\hline \multirow[t]{2}{*}{ OGLE-2006-BLG-265S } & 0.06 & 0.12 & 0.14 & 0.14 & 0.07 & 0.04 & 0.14 & 0.08 & 0.07 & 0.04 & 0.43 & 0.21 & 0.08 \\
\hline & $N_{\mathrm{Fe} \text { I }}$ & $N_{\mathrm{O}}$ & $N_{\mathrm{Na}}$ & $N_{\mathrm{Mg}}$ & $N_{\mathrm{Al}}$ & $N_{\mathrm{Si}}$ & $N_{\mathrm{Ca}}$ & $N_{\mathrm{Ti}}$ & $N_{\mathrm{Cr}}$ & $N_{\mathrm{Ni}}$ & $N_{\mathrm{Zn}}$ & $N_{\mathrm{Y}}$ & $N_{\text {Ba }}$ \\
\hline OGLE-2009-BLG-076S & 57 & 3 & 1 & 5 & 4 & 15 & 10 & 2 & - & 11 & - & - & 2 \\
\hline MOA-2009-BLG-493S & 80 & 3 & 1 & 6 & 6 & 19 & 10 & 5 & 2 & 21 & 1 & - & 4 \\
\hline MOA-2009-BLG-133S & 68 & 2 & 1 & 4 & 6 & 17 & 10 & 4 & 1 & 15 & - & - & 3 \\
\hline MOA-2009-BLG-475S & 53 & 1 & - & 5 & 3 & 12 & 9 & 2 & - & 14 & - & - & 3 \\
\hline MACHO-1999-BLG-022S & 97 & - & 3 & 1 & 2 & 7 & 16 & 14 & 2 & 21 & - & 2 & 3 \\
\hline OGLE-2008-BLG-209S & 146 & 3 & 4 & 5 & 7 & 27 & 19 & 36 & 10 & 40 & 3 & 4 & 4 \\
\hline MOA-2009-BLG-489S & 114 & 3 & 2 & 6 & 6 & 27 & 13 & 16 & 6 & 37 & 3 & 2 & 4 \\
\hline MOA-2009-BLG-456S & 91 & 3 & 2 & 5 & 6 & 26 & 11 & 7 & 3 & 33 & 3 & - & 2 \\
\hline MOA-2008-BLG-311S & 118 & 3 & 4 & 5 & 5 & 26 & 17 & 14 & 9 & 36 & 1 & 2 & 3 \\
\hline MOA-2008-BLG-310S & 122 & 3 & 4 & 4 & 7 & 27 & 17 & 22 & 13 & 42 & 1 & 4 & 3 \\
\hline OGLE-2007-BLG-349S & 103 & 3 & 4 & 3 & 3 & 23 & 18 & 24 & 9 & 39 & 3 & 5 & 4 \\
\hline MOA-2006-BLG-099S & 119 & 3 & 4 & 4 & 4 & 25 & 18 & 30 & 12 & 37 & 3 & 5 & 4 \\
\hline OGLE-2006-BLG-265S & 92 & 3 & 4 & 2 & 3 & 23 & 16 & 13 & 8 & 30 & 2 & 2 & 3 \\
\hline
\end{tabular}

Notes. ${ }^{(\dagger)}$ No abundances are given for MOA-2009-BLG-259S as the errors in the stellar parameters were too large, and for OGLE-2007-BLG514S we only redeteremined stellar parameters and [Fe/H]. Abundance ratios for OGLE-2007-BLG-514S can be found in Epstein et al. (2010). (\$) Note that the abundance ratios for oxygen have been corrected for NLTE effects according to the empirical formula given in Bensby et al. (2004). 
T. Bensby et al.: Chemical evolution of the Galactic bulge as traced by microlensed dwarf and subgiant stars. II.

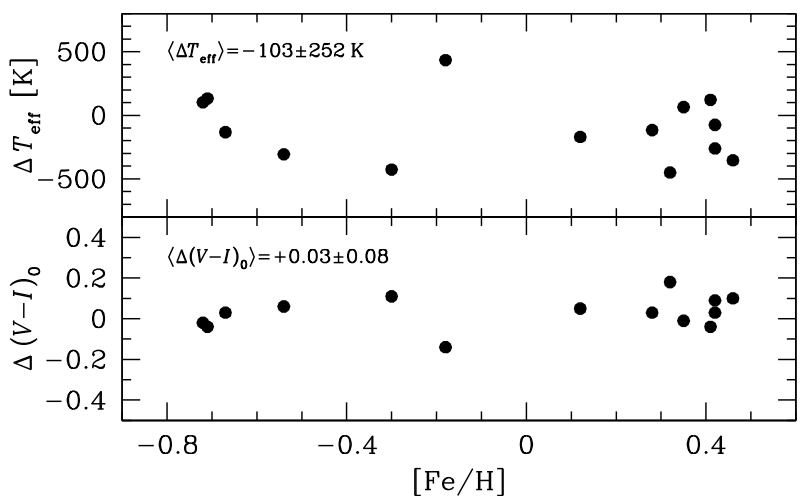

Fig. 5. Top panel shows a comparison of our spectroscopic $T_{\text {eff }}: \mathrm{s}$ to those implied from the colour-[Fe/H] $-T_{\text {eff }}$ calibrations by Ramírez \& Meléndez (2005) versus [Fe/H]. Bottom panel shows a comparison between the colours that our spectroscopic $T_{\text {eff }}$ : $\mathrm{s}$ imply (using the colour$[\mathrm{Fe} / \mathrm{H}]-T_{\text {eff }}$ calibrations by Ramírez \& Meléndez 2005) and the colours based on microlensing techniques. Differences are in both cases given as spectroscopic - photometric.

\section{Metallicity distributions of dwarfs and giants}

The most recent spectroscopic study of a large homogeneous sample of giant stars in the Bulge is by Zoccali et al. (2008). Using FLAMES, the multi-fibre spectrograph at the Very Large Telescope, they studied a sample of 521 giant stars at three latitudes in the Bulge: 204 stars in Baade's window at $l \approx-4^{\circ}$; 213 stars at $l \approx-6^{\circ}$; and 104 stars at $l \approx-12^{\circ}$. The $15 \mathrm{mi}^{-}$ crolensed dwarf stars observed so far are all located at similar angular distances from the Galactic centre as Baade's window (see Fig. 2). Therefore, only the 204 stars in the $l=-4^{\circ}$ field from Zoccali et al. (2008) will be used for comparison.

The average metallicity of the 14 microlensed dwarf and subgiant stars in the Bulge (MOA-2009-BLG-259S excluded, see Sect. 4.8) is $\langle[\mathrm{Fe} / \mathrm{H}]\rangle=-0.08 \pm 0.47$. This is in agreement with the average metallicity of the 204 RGB stars in Baade's window that have $\langle[\mathrm{Fe} / \mathrm{H}]\rangle=-0.04 \pm 0.40$. However, when comparing the two distributions, a two-sided Kolmogorov-Smirnow (KS) test gives a significance level of the null hypothesis, that they are drawn from the same distribution, of $30 \%$ (see Fig. 7). Hence, we can not reject the null hypothesis that the MDF for the $14 \mathrm{mi}-$ crolensed dwarf stars and the MDF for the 204 Bulge RGB stars from Zoccali et al. (2008) are identical.

As discussed by, e.g., Santos et al. (2009) it is possible that a systematic shift in $[\mathrm{Fe} / \mathrm{H}]$ between analyses of dwarf stars and giant stars, perhaps by as much as 0.2 dex, exists. However, the difference in the average metallicity between dwarf and giant stars is essentially zero. If there was a real difference of 0.2 dex between the dwarf stars and the giant stars, how many dwarf stars would we need to observe in order to statistically measure that difference?

In order to estimate the number of stars required to reject the null hypothesis, that the MDF for the sample of 204 RGB stars, $S_{\text {RGB }}$, is not different from the MDF for the observed dwarf stars, we will use a non-parametric bootstrap method. First, we con-

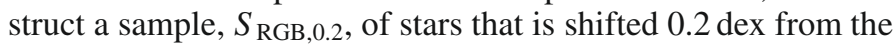
original RGB sample, $S_{\mathrm{RGB}}$. Secondly, we bootstrap $n$ number of stars from sample $S_{\mathrm{RGB}, 0.2}$, thus creating a new sample, $S_{n, 0.2}$, that contains $n$ stars. Next, we perform a two-sided KS-test between samples $S_{\mathrm{RGB}}$ and $S_{n, 0.2}$. If the KS-test yields that the distributions are not the same at the $95 \%$ confidence level, we reject the null hypothesis. We repeat this process $i=10000$ times and take the average, $\langle p\rangle$, of $p_{i}$, where $p_{i}=1$ if the

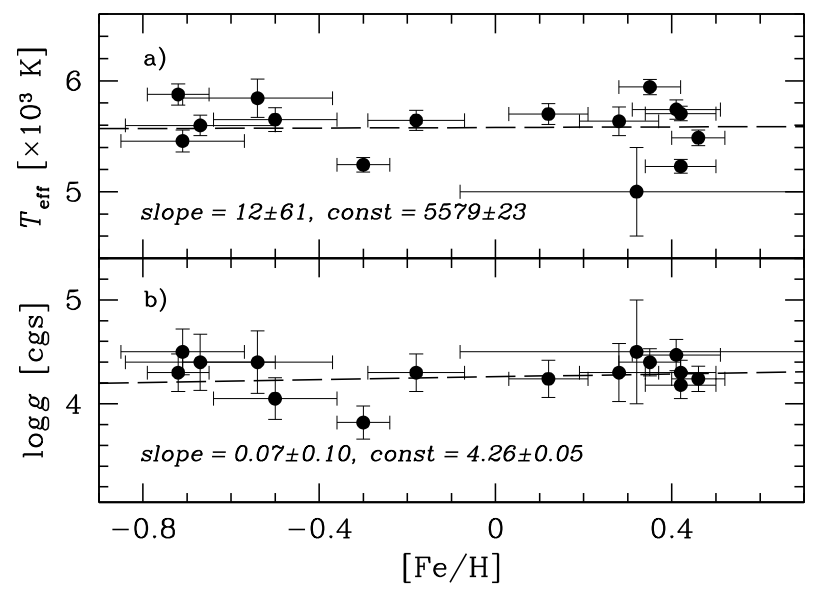

Fig. 6. Effective temperature and surface gravity versus $[\mathrm{Fe} / \mathrm{H}]$. In each figure the regression parameters (slope and constant) are given, as well as their uncertainties. The error bars in the stellar parameters represent the total uncertainty (see Table 2 and Sect. 4.3).

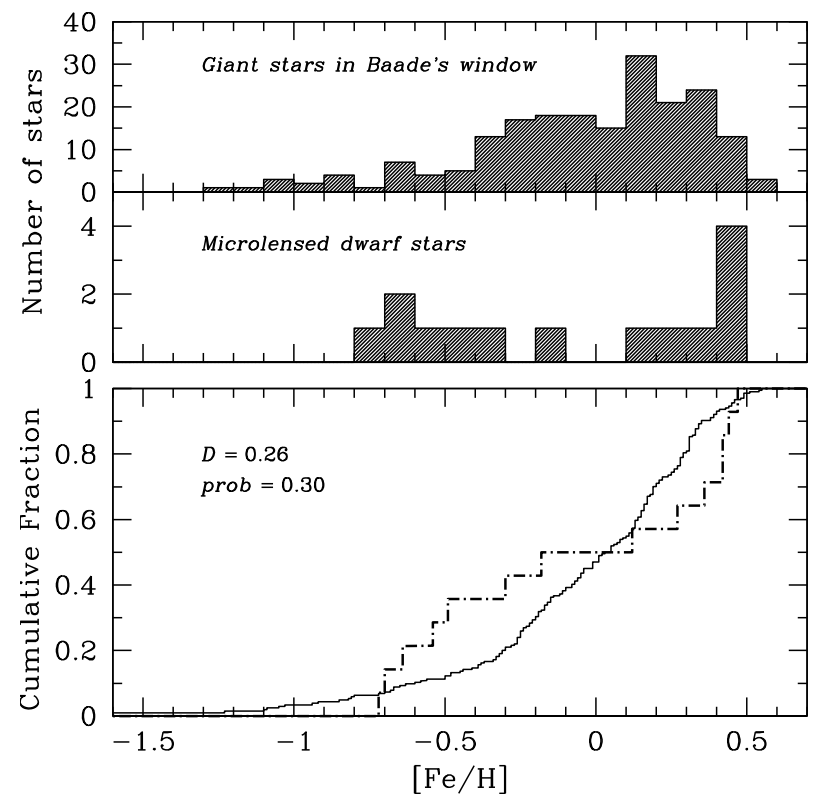

Fig. 7. The top panel shows the MDF for the 204 giant stars in Baade's window from Zoccali et al. (2008), and the middle panel the MDF for the fourteen microlensed dwarf stars (MOA-2009-BLG-259S excluded). Their cumulative metallicity distributions (CMDF) are shown in the bottom panel (giant CMDF marked by solid line, and the dwarf star CMDF by dash-dotted line). The two-sample Kolmogorov-Smirnov $D$ statistic (maximum vertical distance between two distributions) and the corresponding significance level, prob, of $D$ are indicated.

null hypothesis was rejected, and 0 otherwise (type II error). Thus, $\langle p\rangle$ is our probability to identify an intrinsic difference of 0.2 dex in the mean in the MDF for RGB and microlensed dwarf stars. Figure 8 shows how this probability varies with the number of stars in the hypothetical dwarf star sample. In order to statistically verify a difference of at least 0.2 dex, at the $95 \%$ level, we need to observe around 40 stars. Also in Fig. 8 we show how the probability varies if we want to verify a difference of only 0.1 dex between dwarfs and giants. Note that the detection of such a small difference would require the observation of many more microlensed dwarf stars.

Above we shifted the entire MDF by 0.1 and 0.2 dex and did not consider other statistical parameters that describe the MDF 
such as variance, skewness and kurtosis. However, these parameters will most likely make it easier to reject the null hypothesis, that the distributions are the same, if they are considered in the test. Additionally, we only considered 0.1 and 0.2 dex as a difference between the samples. A larger difference will also make it easier to reject the null hypothesis. Our estimate of $\langle p\rangle$ is therefore a lower limit.

Additionally, the average metallicity for the 14 microlensed dwarf stars is in agreement with the average metallicity of the RGB stars. The question is then, what is the significance of this result, given the low number statistics of the microlensed dwarf stars, i.e., can we rule out a shift of 0.2 dex between the MDF for the RGB stars and the MDF for the dwarf stars? We estimate the significance of the agreement between the average metallicities using $10^{5}$ Monte Carlo realisations. In each realisation, we draw 14 stars randomly from the RGB sample. We determine the significance of the agreement between the average of the metallicities by computing the fraction of realisations that fail to produce a dwarf star average metallicity lower or equal to the average metallicity of the RGB stars. Next, we shift the MDF for the RGB stars by a small positive amount, $\delta$, and repeat the above given exercise. Figure 8, lower panel, shows the significance as a function of $\delta$. We find that for $\delta \sim 0.2$, the significance has dropped to 0.05 which indicates that we can rule out the possibility that there is a systematic shift in $[\mathrm{Fe} / \mathrm{H}]$ of $0.2 \mathrm{dex}$ between the MDF for the RGB stars and the MDF for the dwarf stars. However, we need to put this in relation to the standard deviation of the difference between the averages given by

$\sigma_{\mathrm{d}}=\sqrt{\frac{\sigma_{1}^{2}}{n_{1}}+\frac{\sigma_{2}^{2}}{n_{2}}}$

where subscript 1 and 2 indicate the RGB star sample and dwarf star sample, respectively, $\sigma$ is the sample standard deviation, and $n$ is the number of elements in each sample. We find a $\sigma_{\mathrm{d}}$ of 0.12 , which is not even $2 \sigma$ from 0.2 dex. Thus, given the small number of dwarf stars in our sample, $n_{2}=14$, we can not rule out a possible systematic shift of 0.2 dex between the MDF for the RGB stars and the MDF for the dwarf stars, even though the distributions at this point have the same average metallicity. Thus, more microlensed events are needed for the agreement between the averages to be significant.

The above discussion focused on a systematic offset between the dwarf and giant abundances, caused either by systematic effects in the data analysis or differences in the surface compositions of dwarfs and giants because of their differing evolutionary states. However, instead of an overall shift, the dwarf MDF may be different from the giant MDF only at the high metallicity end. Kalirai et al. (2007) suggested that up to 40 per cent of the stars at the metallicity of NGC $6791([\mathrm{Fe} / \mathrm{H}]=$ +0.3 ) skip the He burning phase, resulting in a depletion of the $\mathrm{HB}$ and AGB phases. Therefore, an MDF based on giants may not reflect the MDF for the dwarf stars (i.e. there are metal rich stars missing in the MDF for the RGB stars). The question is then, how many microlensed dwarf stars are required to reject the null hypothesis that the MDF for these dwarfs, that has an excess of metal-rich stars compared to $S_{\mathrm{RGB}}$, is no different than the MDF for $S_{\mathrm{RGB}}$ ? Based on this, we construct a dwarf star sample with more metal-rich stars than $S_{\mathrm{RGB}}, S_{\mathrm{RGB}, \text { Kalirai }}$, under the assumption that $100 \%$ of the dwarfs with $[\mathrm{Fe} / \mathrm{H}]<0.0$ evolve to RGBs, but that the number of dwarfs that evolve to RGBs decrease linearly down to $60 \%$ at $[\mathrm{Fe} / \mathrm{H}]=+0.3$. We extrapolate this linearly for more metal-rich stars, i.e. going down to $\sim 34 \%$ at $[\mathrm{Fe} / \mathrm{H}]=+0.5$. Thus, we add stars, more metal-rich
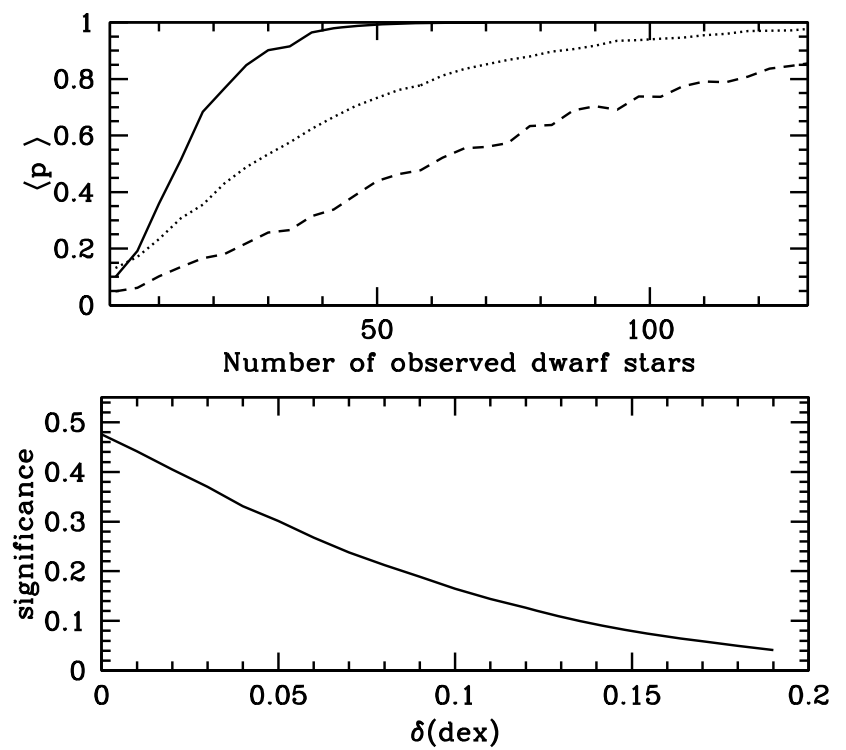

Fig. 8. Upper panel: probability of detecting a difference between the Bulge RGB MDF and the microlensed dwarf MDF as a function of the number of stars in the dwarf sample. Solid and dashed line indicate the probability if the difference is 0.2 and $0.1 \mathrm{dex}$, respectively, in the mean of the MDFs. Dotted line indicates the probability if the difference is that the microlensed dwarf MDF has more metal rich, $[\mathrm{Fe} / \mathrm{H}]>0.0$, stars (i.e., a more symmetric MDF) than the RGB MDF. Lower panel: the significance of the agreement between the average of the metallicities of the RGB MDF and microlensed dwarf MDF, as a function of a speculated (still undetected) difference.

than $[\mathrm{Fe} / \mathrm{H}]=0.0$, to $S_{\mathrm{RGB}}$ to create a hypothetical sample of dwarf stars in the Bulge, $S_{\text {RGB,Kalirai }}$. Additionally, we add stars, drawn from a Gaussian distribution centred on $[\mathrm{Fe} / \mathrm{H}]=+0.2$ with $\sigma=0.4$, to $S_{\mathrm{RGB} \text {,Kalirai }}$ for the metal rich region where there are no observed RGB stars. Thus, making the sample more symmetric. The dotted line in Fig. 8 shows how $\langle p\rangle$ varies for this analysis with the number of stars in $S_{\text {RGB,Kalirai }}$. We note that about 100 stars are required in order to verify that $S_{\mathrm{RGB}}$ is different than $S_{\text {RGB,Kalirai }}$.

Also, there has been some claims that the microlensing event itself alters the spectrum of the source star, and that this is the reason for the very high metallicities of some of the dwarf stars in the Bulge (e.g. Zoccali et al. 2008). Recently, Johnson et al. (2009) investigated the effect that differential limb darkening has on abundance analysis of microlensed dwarf stars. They do find changes in the measured equivalent widths as a result of the differential limb darkening. However, the effect is very small, leading to changes in $T_{\text {eff }}$ less than $45 \mathrm{~K}, \log g$ less than $0.1 \mathrm{dex}$, and $[\mathrm{Fe} / \mathrm{H}]$ less than 0.03 dex. Hence, a possible differential limb darkening can not be responsible for the MDF discrepancy (if any) between dwarf and giant stars.

In summary, it is evident that the extremely super-metalrich MDF proposed by Cohen et al. (2009), exclusively based on dwarf stars with super-solar $[\mathrm{Fe} / \mathrm{H}]$, has shifted toward lower metallicities. The MDF of the 14 microlensed dwarf stars is still poorly determined, currently being double peaked with excesses of low- and high-metallicity stars. Whether this is an effect of small number statistics or not is unclear. More microlensed events will certainly help to clarify the dwarf star MDF and to refine the comparison with the giant star MDF. Also, an outstanding issue is the puzzle presented in Cohen et al. (2010) of the correlation between $A_{\max }$ and $[\mathrm{Fe} / \mathrm{H}]$, which we hope to diagnose as future events are observed. 
T. Bensby et al.: Chemical evolution of the Galactic bulge as traced by microlensed dwarf and subgiant stars. II.

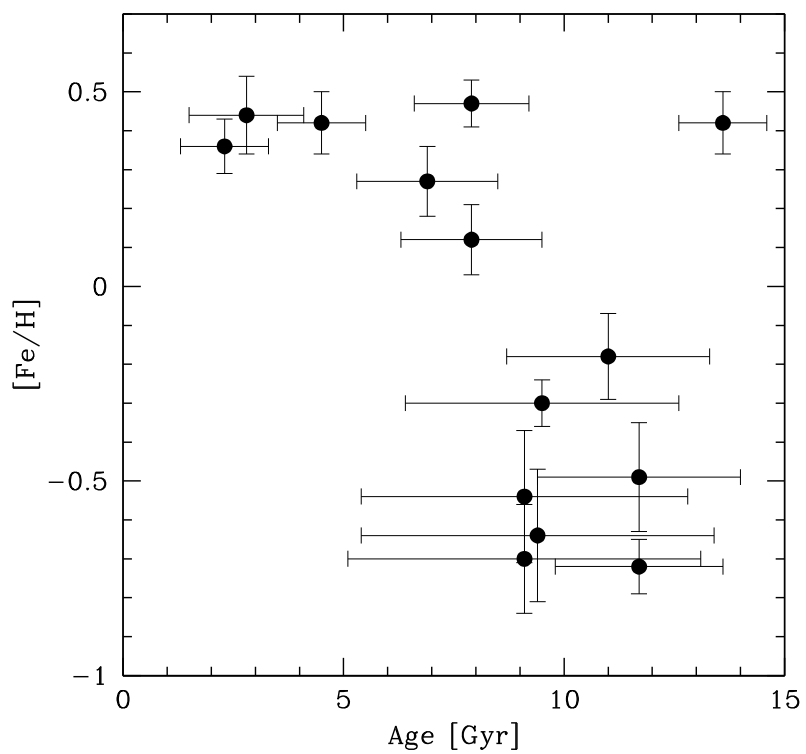

Fig. 9. Ages versus $[\mathrm{Fe} / \mathrm{H}]$ for 14 microlensed dwarf and subgiant stars in the Bulge (MOA-2009-BLG-259S has been excluded). The error bars represent the total uncertainty $[\mathrm{Fe} / \mathrm{H}]$ and age (see Table 2 and Sects. 4.3 and 4.4).

\section{Ages and metallicities}

The 14 microlensed dwarf and subgiant stars (MOA-2009-BLG259S excluded, see Sect. 4.8) in the Bulge have an average age of $8.4 \pm 3.3$ Gyr. Figure 9 shows the age-metallicity diagram and it is evident that stars with sub-solar $[\mathrm{Fe} / \mathrm{H}]$ all have high ages, while at super-solar $[\mathrm{Fe} / \mathrm{H}]$ there is a large spread in age, covering the whole age-range seen in the Galactic disc(s) (e.g., Twarog 1980; Feltzing et al. 2001). That the large age-range seen for super-solar $[\mathrm{Fe} / \mathrm{H}]$ is real is exemplified by two stars: OGLE2007-BLG-349S and MOA-2008-BLG-310S. The first star has a high age while the second has a low age. That the ages are robust can be seen in Fig. 4 and we also note that their stellar parameters have small errors and are derived from a large number of Fe I and Fe II lines (see Table 2 and Fig. 3).

In spite of the small sample, it is notable that we only see the low ages for the metal-rich stars while all stars with sub-solar $[\mathrm{Fe} / \mathrm{H}]$ are consistent with the classical view of the Bulge as an old population (e.g., Holtzman et al. 1993; Feltzing \& Gilmore 2000; Zoccali et al. 2003). As the stars with sub-solar [Fe/H] also have enhanced levels of $\alpha$-elements (see Fig. 10), these stars all appear to adhere to the classical picture of the Bulge as a stellar population that formed rapidly early in the history of the Galaxy (see, e.g., models and discussions in Matteucci 2001).

Overall, the evidence for young stars in the Bulge is scarce. For instance, the extremely deep CMD of $\sim 180000$ field stars in the Bulge by Sahu et al. (2006) show no traces of a young population. It is therefore surprising to find three (MOA-2008BLG-310S, MOA-2008-BLG-311S, and MOA-2006-BLG099S) out of 14 stars to have young ages. At this point we can only speculate on their origin. One interpretation would be that the older stars are all bona fide Bulge stars while the young, metal-rich stars are disc interlopers. In the Galactic disc a young age and a high metallicity is common (e.g., Twarog 1980; Feltzing et al. 2001; Nordström et al. 2004). Also, the innermost Galactic disc is expected to be more metal-rich than the Solar neighbourhood (e.g. Colavitti et al. 2009). However, we still do not now if it is supposed to be young too.
It should furthermore be noted that these young Bulge stars are not brighter than the main old turnoff, they are just too blue to fall on old isochrones (see Fig. 4). Also, there are some theoretical limitations of the isochrone fitting method. First, isochrones at $[\mathrm{Fe} / \mathrm{H}]>+0.3$ have very few calibrators, and, second, the colour of the main sequence is strongly affected by the Y (helium) content, which for the Bulge, or any population with such high metallicity, is poorly known. However, we find a whole range of ages at high metallicities, so we don't see a bias in our ages.

\section{Abundance trends in the Bulge}

\subsection{General appearance}

Figure 10 shows the abundance results for 13 of the 15 the microlensed dwarf and subgiant stars in the Bulge (MOA-2009BLG-259S were excluded, see Sect. 4.8, and for the Epstein et al. (2010) star OGLE-2007-BLG-514S we only determined stellar parameters and $\mathrm{Fe}$ abundances.).

Regarding the $\alpha$-elements $(\mathrm{Mg}, \mathrm{Si}, \mathrm{Ca}$, and $\mathrm{Ti})$, the Bulge dwarfs show enhanced $[\alpha / \mathrm{Fe}]$ ratios at sub-solar $[\mathrm{Fe} / \mathrm{H}]$, that decline when approaching solar metallicities. At higher metallicities the $[\alpha / \mathrm{Fe}]$ ratios are around or slightly higher than solar. The oxygen trend is similar to the $\alpha$-element trends at sub-solar metallicities, but differs at super-solar $[\mathrm{Fe} / \mathrm{H}]$ where it continues to decrease. The oxygen abundances that are based on the infrared triplet lines at $773 \mathrm{~nm}$ have been NLTE corrected the according to the empirical formula given in Bensby et al. (2004).

Generally, abundance trends of the dwarf stars in the Bulge are very well-defined. The scatter in the $[\mathrm{Ti} / \mathrm{Fe}]-[\mathrm{Fe} / \mathrm{H}]$ plot for instance is remarkably low. The canonical interpretation of the plateau of high $\alpha$-element abundances relative to iron at low metallicities is due to early and rapid chemical enrichment of the Bulge by massive stars. When these stars die, they explode as core-collapse supernovae, producing a lot of $\alpha$-elements relative to iron. At some point low-mass stars start to contribute to the chemical enrichment, and since these produce less of the $\alpha$-elements the $[\alpha / \mathrm{Fe}]$ ratio will start to decline.

The enhanced $[\mathrm{Na} / \mathrm{Fe}] \approx+0.1$ for metal-rich disc stars was already noticed by Edvardsson et al. (1993), Feltzing \& Gustafsson (1998), Shi et al. (2004), and also in Bensby et al. (2005) an upturn in $[\mathrm{Na} / \mathrm{Fe}]$ can be seen. Our microlensed dwarf stars are in full agreement with these disc results.

The $[\mathrm{Ba} / \mathrm{Fe}]$ trend is flat and slightly under-abundant compared to the Sun for all $[\mathrm{Fe} / \mathrm{H}]$.

\subsection{Comparisons to the Galactic thick disc}

In Fig. 10 we also show the thick disc abundance trends based on dwarf stars in the Solar neighbourhood (taken from Bensby et al. 2003, 2005, and Bensby et al., in prep.). These thick disc stars have been analysed using the exact same methods (spectral line lists, atomic data, model stellar atmospheres, etc.) that we use for the microlensed dwarf stars. Hence, any differences between the stars from the two stellar populations should be real, and not due to unknown systematic effects.

The first thing that can be taken away from Fig. 10 is that, at sub-solar metallicities, the abundance trends for the Bulge dwarf stars are very similar to those of the thick disc stars. The solid lines shown in the figures indicate the median abundance ratio as a function of metallicity for thick disc stars. Even though the appearance of the abundance trends at sub-solar $[\mathrm{Fe} / \mathrm{H}]$ are very similar between the Bulge and the thick disc, it is also 

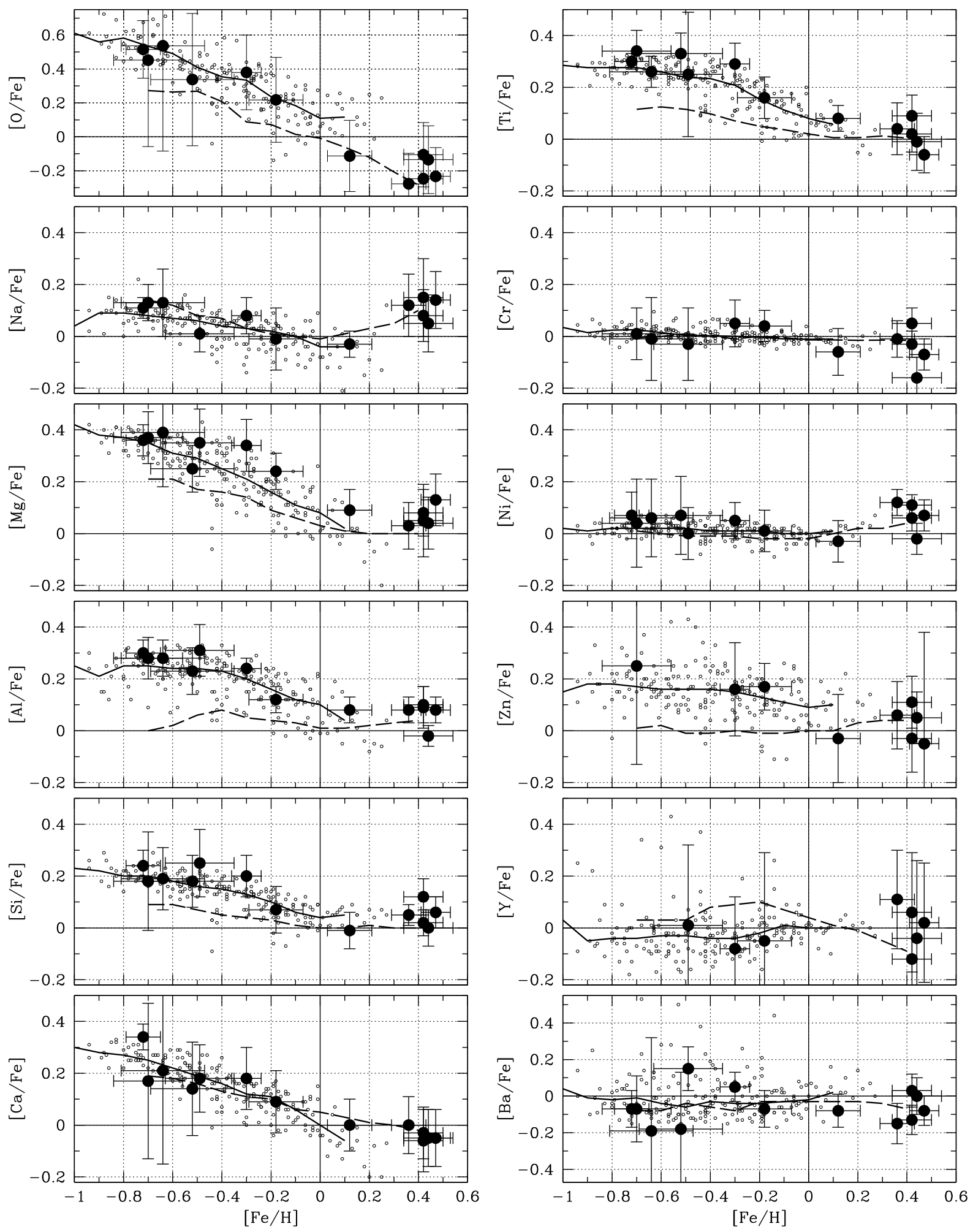

Fig. 10. Abundance results for 13 microlensed dwarf and subgiant stars in the Bulge (marked by filled bigger circles). Thick disc stars from Bensby et al. (2010, in prep.) are shown as small circles, and the solid line is the running median of the shown thick disc sample, and the dashed line the running median of the (not shown) thin disc sample from Bensby et al. (2010, in prep.). The error bars represent the total uncertainty in the abundance ratios (see Sect. 4.3 and Table 5).

evident that for many elements the Bulge stars appear to be slightly more enhanced than the median thick disc. The Bulge stars seem to occupy the upper envelope of the thick disc abundance trends. Part of this apparent shift in the abundance trends between the two populations could be due to that the thick disc sample is kinematically selected, and hence will unavoidably be mixed, to some degree, with kinematically hot thin disc stars (see Bensby et al. 2007). The median thick disc line that we show in Fig. 10 will then be slightly too low. However, this effect should only be important when approaching solar metallicities, 


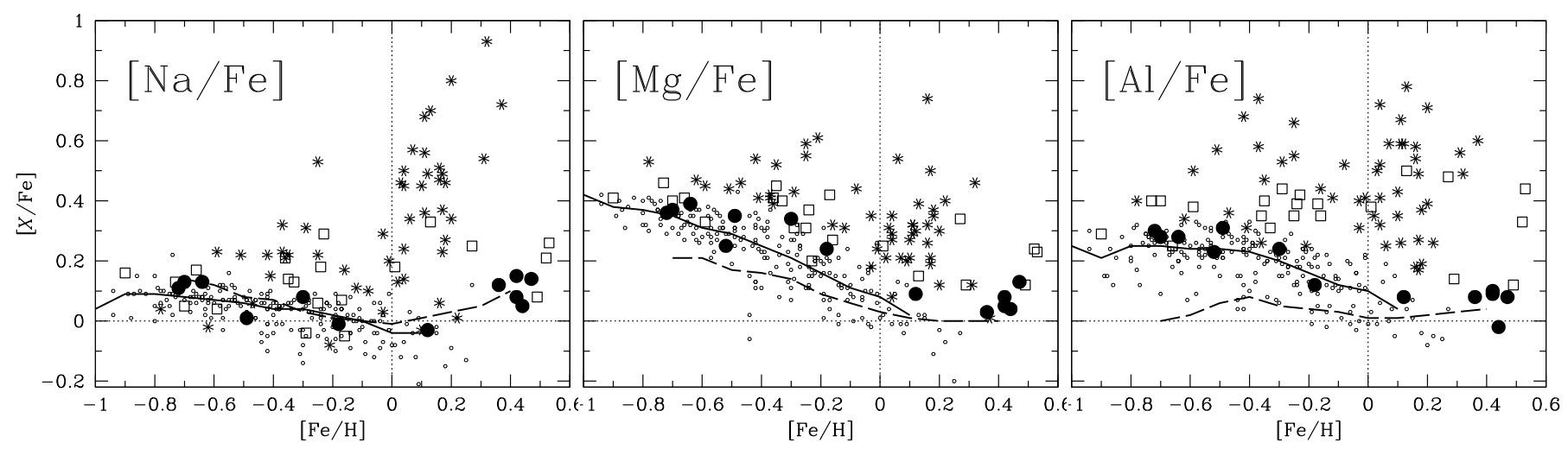

Fig. 11. Comparisons of $[\mathrm{Na} / \mathrm{Fe}],[\mathrm{Mg} / \mathrm{Fe}]$, and $[\mathrm{Al} / \mathrm{Fe}]$ between our microlensed dwarf stars (filled circles), giants from Fulbright et al. (2007) (empty squares), giants from Lecureur et al. (2007) (asterisks), nearby thick disc stars from Bensby et al. (in prep.) (small open circles). Solid and dashed lines represent the running median of the thick and thin disc stars, respectively (same as in Fig. 10).

and we do see a shift between the Bulge and the thick disc at lower $[\mathrm{Fe} / \mathrm{H}]$ as well, where the kinematic confusion between the thin and thick discs should be negligible. However, the shift is not for all elements, and it is small, on the order of 0.05 dex or less. More microlensing events will help us to clarify if this shift is real or not.

A possible link between the Bulge and the Galactic thick disc based on similarities of abundances was first suggested by Prochaska et al. (2000), pointing out the "excellent agreement" between the abundance ratios in their sample of ten thick disc stars to those of the Bulge giants of McWilliam \& Rich (1994). As the thick disc sample of Prochaska et al. (2000) only reached $[\mathrm{Fe} / \mathrm{H}] \approx-0.4$, the downturn in $[\alpha / \mathrm{Fe}]$ that we now see in the thick disc at $[\mathrm{Fe} / \mathrm{H}] \approx-0.35$ (e.g. Feltzing et al. 2003; Bensby et al. 2007) was at that time not known. Hence, when later studies of giant stars in the Bulge showed that the $[\alpha / \mathrm{Fe}]$ remained high even at super-solar metallicities (e.g. Fulbright et al. 2007), in contrast to the declining thick disc trends, the possible connection between the Bulge and the thick disc became less clear. It should also be noted that Prochaska et al. (2000) did not analyse both the Bulge and thick disc samples. Also, recently, Meléndez et al. (2008) presented a consistent analysis of giant stars in both the Bulge and the thick disc that found a similarity between them for C, N, O (recently confirmed by Ryde et al. 2009). The agreement is extended to other $\alpha$-elements in the upcoming study by Alves-Brito et al. (2010). However, the first confirmation that the Bulge and the thick disc have similar abundance patterns based on dwarf stars came from Bensby et al. (2009b,a), and with this study it now appears well established that the Bulge and the thick disc have had, to some degree, similar histories.

Before interpreting the apparent agreement between the Bulge and thick disc abundance trends, it is important to recognise that the MDFs for the thick disc and the Bulge differ in the average as well as width of their MDFs. The metallicity distribution for the thick disc peaks at $[\mathrm{Fe} / \mathrm{H}] \approx-0.6$ (e.g., Carollo et al. 2010), and its metal-rich tail likely reach solar metallicities (Bensby et al. 2007). Stars with $[\mathrm{Fe} / \mathrm{H}]>0$ that can be kinematically classified as thick disc stars, are heavily biased to belong to the high-velocity tail of the thin disc (Bensby et al. 2007). Even though the average metallicity of the Bulge and the shape of its MDF is under debate (see Sect. 5) it is clear that it spans the full range of metallicities of the thick disc, and in addition reaches very high, super-solar $[\mathrm{Fe} / \mathrm{H}]$. Hence, the agreement, as regards the abundance trends, between the Bulge and the thick disc can currently only be established for sub-solar metallicities. The comparison that can be made at super-solar $[\mathrm{Fe} / \mathrm{H}]$ is between the Bulge stars and thin disc stars (dashed lines in Fig. 10) that reach similar high metallicities. As the abundance trends in Fig. 10 show, the agreement between the Bulge and the thin disc at $[\mathrm{Fe} / \mathrm{H}]>0$ is good. Looking at, e.g., the $[\mathrm{Na} / \mathrm{Fe}],[\mathrm{Ni} / \mathrm{Fe}]$, and $[\mathrm{Ba} / \mathrm{Fe}]$ trends, which are not simply flat at the highest $[\mathrm{Fe} / \mathrm{H}]$, the Bulge stars nicely extend the trends that are seen for the nearby thin disc stars. However, Epstein et al. (2010) found a [Ba/Fe] value noticeably below the disc for OGLE-2009-BLG$514 \mathrm{~S}$. The Ba II lines are strong and very sensitive to the microturbulence parameter, and therefore measuring the abundances of another heavy element, such as La, would be a useful check.

With this study where we compare Bulge dwarf stars with disc $d$ warf stars, and with the recent studies by Meléndez et al. (2008) and Alves-Brito et al. (2010), all using internally consistent methods, it appears clear that the Bulge and thick disc abundance trends are similar. The agreement between the Bulge and the thick disc means that conclusions from recent theoretical works, developed under the assumptions that the abundance trends in the Bulge and thick disc are different, may not be valid.

We caution that the comparisons that we do and that have been done by others are between the Bulge stars close to the Galactic centre and thin and thick disc stars in the vicinity of the Sun, i.e., at a distance of approximately $8 \mathrm{kpc}$ from the Galactic centre. In order verify a possible connection between the Bulge and the Galactic thick (and thin) disc(s) we need disc stellar samples much closer to the Bulge, say at $4 \mathrm{kpc}$ from the Galactic centre. At that distance the contamination of Bulge stars in a disc stellar sample should be small, allowing us to directly compare the populations. If, for instance, the star formation has been more rapid in the inner disc than in the Solar neighbourhood, or in the outer disc, we should expect the "knee" in the $\alpha$-element abundance trends at higher $[\mathrm{Fe} / \mathrm{H}]$ than what we see in the Solar neighbourhood. Then that could explain the apparent slight shift in the abundance trends between the Bulge and the thick disc in the Solar neighbourhood that we see. However, no such inner disc sample is currently available.

\subsection{Comparisons to Bulge giants}

It should be noted that some recent studies of Bulge giant stars claim that the Bulge is enhanced in the $\alpha$-elements with respect 

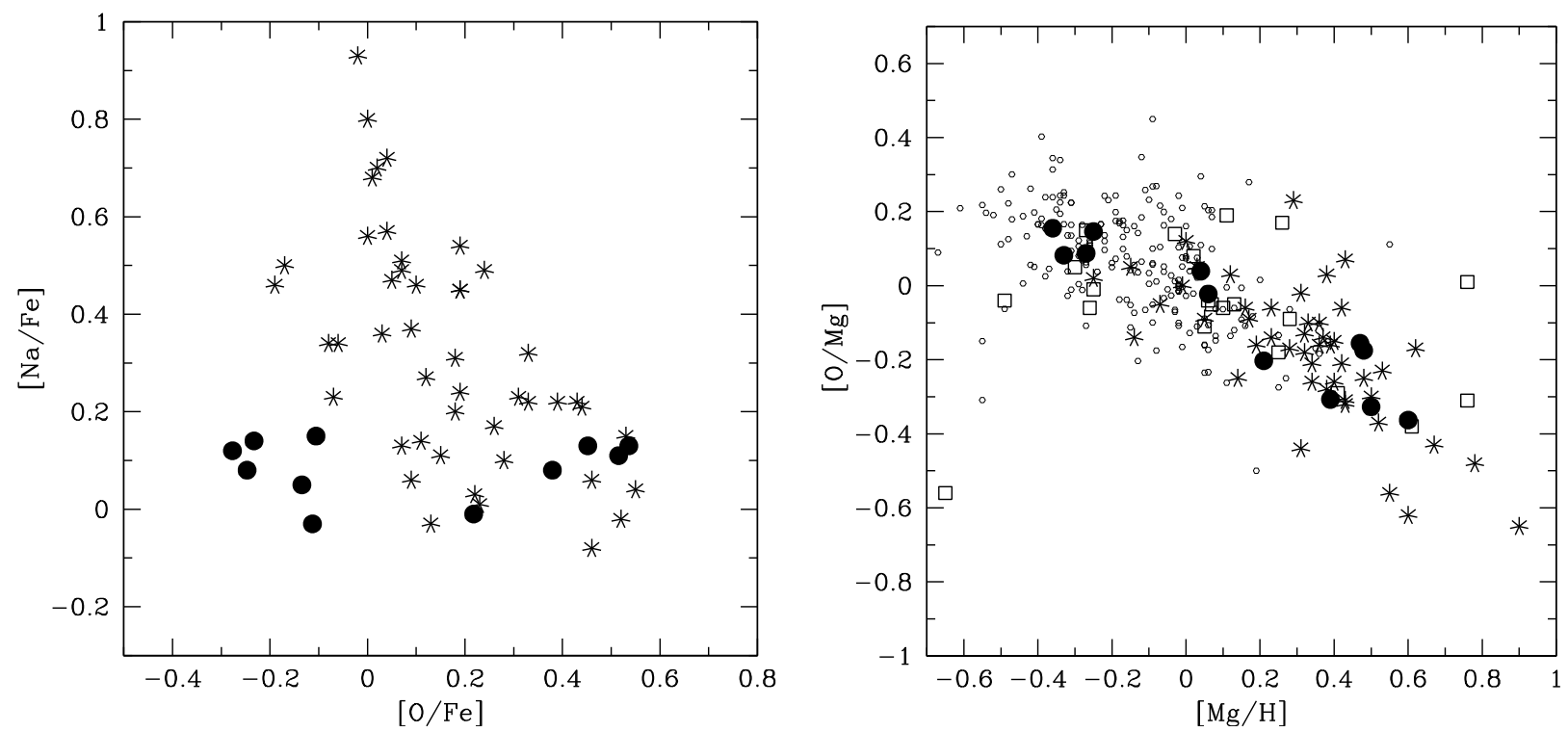

Fig. 12. $[\mathrm{Na} / \mathrm{Fe}]$ versus $[\mathrm{O} / \mathrm{Fe}]$ (left panel) and $[\mathrm{O} / \mathrm{Mg}]$ versus $[\mathrm{Mg} / \mathrm{H}]$ (right panel) for our microlensed dwarf stars (filled circles), the giants from Fulbright et al. (2007) (open squares), the giants from Lecureur et al. (2007) (asterisks), and thick disc dwarf stars in the Solar neighbourhood (Bensby et al. 2003, 2005, and in prep.) (small open circles).

to the stars of the Galactic disc. For instance, Rich \& Origlia (2005) compare their Bulge giants to nearby thin disc giants and find the Bulge ones to be more enhanced; Zoccali et al. (2006); Fulbright et al. (2007) and Lecureur et al. (2007) find their Bulge giants to be more enhanced than comparison samples of nearby thin and thick disc dwarf stars. In contrast, the recent study by Meléndez et al. (2008), and the upcoming study by Alves-Brito et al. (2010), compare Bulge giants with nearby thick disc giants, and find the Bulge and thick disc abundance trends to be similar.

In Fig. 11 we compare $[\mathrm{Na} / \mathrm{Fe}],[\mathrm{Mg} / \mathrm{Fe}]$ and $[\mathrm{Al} / \mathrm{Fe}]$ between our microlensed Bulge dwarfs, the giants from Fulbright et al. (2007) and Lecureur et al. (2007), and the Solar neighbourhood thin and thick disc dwarfs from Bensby et al. (2003, 2005, and in prep.). It is evident that the giants from Lecureur et al. (2007) are much more enhanced in $\mathrm{Na}, \mathrm{Mg}$, and $\mathrm{Al}$ than any of the other comparison samples, with our dwarf stars having lower abundances, the Fulbright et al. (2007) giants having intermediate abundances, and the Lecureur et al. (2007) giants having the highest abundances. The higher abundances of the giant stars might be due to the way these abundances have been normalised to the Sun. For dwarf stars with spectral types similar to the Sun, the normalisation is straightforward, it is just to analyse the Sun in the same way and then subtract the solar abundance. However, for giants, having very different $\log g$ : $\mathrm{s}$ and $T_{\mathrm{eff}}: \mathrm{s}$ than the Sun, the normalisation is usually done to another standard star, such as, e.g., $\mu$ Leo. This could partly explain the levels of the abundances, progressing from our dwarf stars, to the Fulbright et al. (2007) giants, and to the Lecureur et al. (2007) giants.

Looking closer at the Lecureur et al. (2007) data it appears as if there is a very well defined lower envelope just above the thick disc trend, and on top of that a large scatter of stars with higher abundance ratios, spreading upwards. We suspect that this might be due to line blending, and possibly lack of accurate continuum points, in the extremely crowded spectra that metal-rich giants have. The lower envelope that is seen in the Lecureur et al. (2007) is what could be expected if blending is the case. A number of stars that have less (or very few) blends forming the well-behaved lower envelope of the trends, while others are more affected, leading to randomly increased equivalent widths, and hence randomly increased abundances.

\subsection{No Na-O anti-correlation}

The Na-O anti-correlation that has seen in all globular clusters studied so far is not present for field stars (e.g., Carretta et al. 2009). Therefore, one of the most striking results from Lecureur et al. (2007) was the Na-O anti-correlation that they found for their Bulge giants, and they claim it is probably an effect of the Bulge chemical evolution. However, Fig. 12 (left panel) shows the $\mathrm{Na}-\mathrm{O}$ plot for our microlensed dwarf stars and there is no $\mathrm{Na}-\mathrm{O}$ anticorrelation present. Instead $[\mathrm{Na} / \mathrm{Fe}]$ versus $[\mathrm{O} / \mathrm{Fe}]$ is flat. Given the large spreads that Lecureur et al. (2007) see for $\mathrm{Na}, \mathrm{Mg}$, and $\mathrm{Al}$ (see Fig. 11) it appears likely that their results are affected by systematic errors (blending presumably).

\subsection{Metallicity dependent oxygen yields}

In Fig. 12 (right panel) the evolution of $[\mathrm{O} / \mathrm{Mg}]$ vs. $[\mathrm{Mg} / \mathrm{H}]$ is shown, which reveals a very tight correlation. Our results from microlensed dwarfs have significantly less scatter than the corresponding values for Bulge red giants (e.g. Lecureur et al. 2007; Fulbright et al. 2007; Alves-Brito et al. 2010) presumably due to the lesser influence of blends and better determined stellar parameters. The dwarf-based slope is also slightly shallower than for the giants. A declining trend in $[\mathrm{O} / \mathrm{Mg}]$ towards higher metallicity is expected with traditional metallicity-dependent supernovae yields such as those of Woosley \& Weaver (1995) but our slope is steeper, which may signal an metallicity-dependence introduced for example by mass-loss in massive stars (e.g. Maeder 1992; Meynet \& Maeder 2002) as discussed by McWilliam et al. (2008) and Cescutti et al. (2009). These findings are based on the increased Wolf-Rayet stellar wind efficiency at higher metallicity that removes a larger fraction of $\mathrm{He}$ and $\mathrm{C}$ before they are converted to $\mathrm{O}$ and thus decreasing the $\mathrm{O}$ production but leaving the $\mathrm{Mg}$-yield largely unaltered. The similarity between the Bulge and disc results implies that these metallicity-dependent nucleosynthesis yields are a general feature but also argues against substantial differences in the initial mass function (IMF) between the two populations. 
T. Bensby et al.: Chemical evolution of the Galactic bulge as traced by microlensed dwarf and subgiant stars. II.

\section{The origin of the Galactic bulge}

To summarise our observations, we find that the stars with high $[\alpha / \mathrm{Fe}]$ and low-metallicity in the Bulge are old, but the stars with high metallicities and solar (or subsolar) $[\alpha / \mathrm{Fe}]$ ratios span a range of ages from $10 \mathrm{Gyr}$ to $3 \mathrm{Gyr}$. What does this mean for the origin of the Bulge?

The old age, high $[\alpha / \mathrm{Fe}]$ population can be explained through the standard chemical pattern in systems dominated by type II SNe that is common to all stellar systems. That the high $[\alpha / \mathrm{Fe}]$ ratio persist to $[\mathrm{Fe} / \mathrm{H}]>-0.6$ indicates that star formation proceeded very efficiently in the event(s) that created the Bulge. Such events could be early mergers of subhalos which drive efficient star formation as well as contributing their own high $[\alpha / \mathrm{Fe}]$ and low $[\mathrm{Fe} / \mathrm{H}]$ stars (Rahimi et al. 2010), or early fragmentation of the disc into clumps of stars and gas which then rapidly merge to form the Bulge (Immeli et al. 2004). These results may also be consistent with the secular evolution of the disc, depending on the age-metallicity- $[\alpha / \mathrm{Fe}]$ relation present in the inner disc. In the models of Schönrich \& Binney (2009), the inner disc is composed of stars that have old ages and higher $[\alpha / \mathrm{Fe}]$ at the same $[\mathrm{Fe} / \mathrm{H}]$ than stars formed in the thin disc in the Solar neighbourhood. Some of these inner disc stars then migrate outwards to form the local thick disc. Thus it would not be surprising that the Bulge (=puffed up inner disc) stars should be chemically similar to the local thick disc (=migrated inner disc) stars, which is exactly what we see. The model by Schönrich \& Binney (2009) requires a number of assumptions/approximations/parameterisation of the migration and heating processes, and is tuned to explain the thick disc in the Solar neighbourhood, so observational evidence (still missing) of the nature of the inner disc is, once again, important. Our results are not in agreement with the models of Immeli et al. (2004) where the gas cools less efficiently and the instability that forms the bar sets in at later times, because those models predict a decline in $[\alpha / \mathrm{Fe}]$ starting at much lower metallicities (see their Fig. 10).

The solar $[\alpha / \mathrm{Fe}]$ and high metallicity stars that span a range of ages are more of a puzzle. The old stars imply that part of the Bulge got a head start on its chemical evolution, so that some 10 Gyr old stars were formed from a population that was already producing type Ia $\mathrm{SNe}$, while other $10 \mathrm{Gyr}$ stars were forming out of gas that had just been enriched with type II SNe. Old, low $[\alpha / \mathrm{Fe}]$ stars are found in the dwarf spheroid galaxies, but accreting a Sagittarius-like object would not explain the pattern, because the low $[\alpha / \mathrm{Fe}]$ stars in Sagittarius have too low metallicities (e.g., Venn et al. 2004). In the simulations of Rahimi et al. (2010), the bulges experience a series of mergers over a period of $\sim 5$ Gyr, leading to populations of "old", "intermediate" and "young" stars. The distributions of $[\mathrm{Mg} / \mathrm{Fe}]$ for these populations do show some old stars with low $[\mathrm{Mg} / \mathrm{Fe}]$, (as well the expected shift to low $[\mathrm{Mg} / \mathrm{Fe}]$ for the younger stars) but they caution that their code suppresses mixing among gas particles, leading to artificially high abundance ratio dispersions. Nonetheless, this is what we see in our data, so perhaps the Galaxy found a way to suppress mixing as well, maybe with a merger history different than the two cases in Rahimi et al. (2010), where two of the subclumps that merged had different starting times for star formation relative to today.

Finally, the younger, low $[\alpha / \mathrm{Fe}]$ and high metallicity stars show that star formation persisted in the components that created the Bulge. These stars are seen in the disc fragmentation models of Immeli et al. (2004), where, for the cold gas model that agrees with the turnover in the $[\alpha / \mathrm{Fe}]$ vs. $[\mathrm{Fe} / \mathrm{H}]$ diagram,
$30 \%$ of the baryons are not rapidly converted to stars. Instead, low level star formation occurs for several Gyr and produces a peak in the $[\mathrm{Mg} / \mathrm{Fe}]$ histogram of the Bulge at $[\mathrm{Mg} / \mathrm{Fe}]=-0.2$. This kind of stars are also seen in the models of Rahimi et al. (2010) where new stars formed after mergers at later times are polluted with type Ia ejecta as well. Finally, depending on the star formation of the thick disc, these stars may be present in that component and then used to make the Bulge.

To distinguish further among these models, the inner disc of the Galaxy needs to be better characterised observationally. In addition, more Bulge dwarfs with accurate ages, metallicities and abundance ratios would help clarify whether the age spread is confined to the higher metallicities and whether the oldest low $[\alpha / \mathrm{Fe}]$ stars are as old as the lower metallicity high $[\alpha / \mathrm{Fe}]$ stars. Finally, the elements produced in type Ia SNe are not the only chemical evolution "clock" available, and measuring elements produced in AGB stars ( $\mathrm{C}, \mathrm{N}$ and s-process), for example, would test whether chemical evolution really began earlier for some stars now in the Bulge than for others.

\section{Summary}

With this study we have doubled the number statistics on the data for microlensed dwarf and subgiant stars in the Bulge. All stars have been observed with high-resolution spectrographs and from a detailed elemental abundance analysis we present results for $\mathrm{O}, \mathrm{Na}, \mathrm{Mg}, \mathrm{Al}, \mathrm{Si}, \mathrm{Ca}, \mathrm{Ti}, \mathrm{Cr}, \mathrm{Fe}, \mathrm{Ni}, \mathrm{Zn}, \mathrm{Y}$, and $\mathrm{Ba}$. The method we utilise is identical to the method used for a large sample of $702 \mathrm{~F}$ and $\mathrm{G}$ dwarf stars in the thin and thick discs in the Solar neighbourhood. Therefore, any differences between the Bulge stars and the disc stars should be genuine, and not due to unknown (systematic) uncertainties. We have also determined stellar ages for the stars, a task that is impossible to do with giant stars.

The main results and conclusions that can be drawn from our sample of 15 microlensed stars in the Bulge are:

1. The stars span a wide range of metallicities between $[\mathrm{Fe} / \mathrm{H}]=-0.72$ up to super-solar metallicity of $[\mathrm{Fe} / \mathrm{H}] \approx$ +0.54 .

2. The mean metallicity of the 14 microlensed dwarf and subgiant stars is $\langle[\mathrm{Fe} / \mathrm{H}]\rangle=-0.08 \pm 0.47$ in good agreement with the 204 giant stars in Baade's window from Zoccali et al. (2008) that have an average metallicity of -0.04 dex. However, a two-sided KS-test gives only a low $30 \%$ probability that microlensed dwarf stars and giant stars in BaadeÕs window have the same MDFs. The low probability is due to the skewed and uneven metallicity distribution of the dwarf stars, with excesses at both low and high metallicities. More observations of microlensed dwarf stars will certainly refine the comparison. It is clear though that the extremely metal-rich MDF for the Bulge that Cohen et al. (2009) propose is not borne out by the larger sample presented here.

3. The abundance trends that the microlensed dwarf stars show are surprisingly well-defined. At sub-solar $[\mathrm{Fe} / \mathrm{H}]$ they are more or less coincident with the abundance trends of the Galactic thick disc as traced by nearby dwarf stars (Bensby et al. 2003, 2005, 2007). At super-solar [Fe/H] they follow the trends we see for nearby thin disc dwarf stars. However, due to the high ages that some of the Bulge stars possess at super-solar $[\mathrm{Fe} / \mathrm{H}]$, and due to the lack of Bulge stars at sub-solar $[\mathrm{Fe} / \mathrm{H}]$ with thin disc abundance ratios we see no obvious connection between the Bulge and the thin disc. 
4. All stars with sub-solar $[\mathrm{Fe} / \mathrm{H}]$ are old (around $10 \mathrm{Gyr}$ ) and have high $[\alpha / \mathrm{Fe}]$ ratios, consistent with fast enrichment by core-collapse supernovae during the early stages of the formation of the Galaxy. At super-solar $[\mathrm{Fe} / \mathrm{H}]$ we have a few old stars but also three stars with ages lower than 5 Gyr. This is inconsistent with, e.g., recent CMDs of field stars in the Bulge based on deep imaging with HST/ACS, that show no evidence for a young stellar component in the Bulge. The average age for our sample of microlensed dwarf stars is $8.4 \pm 3.3$ Gyr.

5. Additionally, our results indicate that the red clump stars in the Bulge have $(V-I)_{0}=1.08$.

Based on these results and conclusions we speculate on the origin of the Bulge and we must conclude that it is still poorly constrained.

Acknowledgements. S.F. is a Royal Swedish Academy of Sciences Research Fellow supported by a grant from the Knut and Alice Wallenberg Foundation. Work by A.G. was supported by NSF Grant AST-0757888. A.G.-Y. is supported by the Israeli Science Foundation, an EU Seventh Framework Programme Marie Curie IRG fellowship and the Benoziyo Center for Astrophysics, a research grant from the Peter and Patricia Gruber Awards, and the William Z. and Eda Bess Novick New Scientists Fund at the Weizmann Institute. A.U. acknowledges support by the Polish MNiSW grant N20303032/4275. S.L. research was partially supported by the DFG cluster of excellence "Origin and Structure of the Universe". J.M. is supported by a Ciência 2007 contract, funded by FCT/MCTES (Portugal) and POPH/FSE (EC) and he acknowledges financial support from FCT project PTDC/CTE-AST/098528/2008. T.S. acknowledges support from grant JSPS20740104. D.A. thanks David Bolin at the Centre for Mathematical Sciences (Lund University) for help with statistics. We would like to thank Bengt Gustafsson, Bengt Edvardsson, and Kjell Eriksson for usage of the MARCS model atmosphere program and their suite of stellar abundance (EQWIDTH) programs. We also thank Judy Cohen and Courtney Epstein for providing reduced spectra of their microlensing events. This research has also made use of the Keck Observatory Archive (KOA), which is operated by the W.M. Keck Observatory and the NASA Exoplanet Science Institute (NExScI), under contract with the National Aeronautics and Space Administration.

\section{References}

Alves-Brito, A., Melendez, J., Asplund, M., Ramirez, I., \& Yong, D. 2010, A\&A, in press, [arXiv: $1001.2521 \mathrm{v} 1]$

Asplund, M., Gustafsson, B., Kiselman, D., \& Eriksson, K. 1997, A\&A, 318, 521

Bensby, T., Feltzing, S., \& Lundström, I. 2003, A\&A, 410, 527

Bensby, T., Feltzing, S., \& Lundström, I. 2004, A\&A, 415, 155

Bensby, T., Feltzing, S., Lundström, I., \& Ilyin, I. 2005, A\&A, 433, 185

Bensby, T., Zenn, A. R., Oey, M. S., \& Feltzing, S. 2007, ApJ, 663, L13

Bensby, T., Feltzing, S., Johnson, J. A., et al. 2009a, ApJ, 699, L174

Bensby, T., Johnson, J. A., Cohen, J., et al. 2009b, A\&A, 499, 737

Bond, I. A., Abe, F., Dodd, R. J., et al. 2001, MNRAS, 327, 868

Carollo, D., Beers, T. C., Chiba, M., et al. 2010, ApJ, 712, 692

Carretta, E., Bragaglia, A., Gratton, R., \& Lucatello, S. 2009, A\&A, 505, 139

Cavallo, R. M., Cook, K. H., Minniti, D., \& Vandehei, T. 2003, in SPIE Conf. Ser. 4834, ed. P. Guhathakurta, 66

Cescutti, G., Matteucci, F., McWilliam, A., \& Chiappini, C. 2009, A\&A, 505, 605

Clarkson, W., Sahu, K., Anderson, J., et al. 2008, ApJ, 684, 1110

Cohen, J. G., Huang, W., Udalski, A., Gould, A., \& Johnson, J. A. 2008, ApJ, 682, 1029

Cohen, J. G., Thompson, I. B., Sumi, T., et al. 2009, ApJ, 699, 66

Cohen, J. G., Gould, A., Thompson, I. B., et al. 2010, ApJ, 711, L48

Colavitti, E., Cescutti, G., Matteucci, F., \& Murante, G. 2009, A\&A, 496, 429
Cunha, K., \& Smith, V. V. 2006, ApJ, 651, 491

Dekker, H., D’Odorico, S., Kaufer, A., Delabre, B., \& Kotzlowski, H. 2000, in Optical and IR Telescope Instrumentation and Detectors, ed. M. Iye, \& A. F.

Moorwood, Proc. SPIE, 4008, 534

Demarque, P., Woo, J.-H., Kim, Y.-C., \& Yi, S. K. 2004, ApJS, 155, 667

Edvardsson, B., Andersen, J., Gustafsson, B., et al. 1993, A\&A, 275, 101

Epstein, C. R., Johnson, J. A., Dong, S., et al. 2010, ApJ, 709, 447

Feltzing, S., \& Gustafsson, B. 1998, A\&AS, 129, 237

Feltzing, S., \& Gilmore, G. 2000, A\&A, 355, 949

Feltzing, S., Bensby, T., \& Lundström, I. 2003, A\&A, 397, L1

Feltzing, S., Holmberg, J., \& Hurley, J. R. 2001, A\&A, 377, 911

Frogel, J. A. 1988, ARA\&A, 26, 51

Fulbright, J. P., McWilliam, A., \& Rich, R. M. 2006, ApJ, 636, 821

Fulbright, J. P., McWilliam, A., \& Rich, R. M. 2007, ApJ, 661, 1152

Gustafsson, B., Bell, R. A., Eriksson, K., \& Nordlund, A. 1975, A\&A, 42, 407

Holtzman, J. A., Light, R. M., Baum, W. A., et al. 1993, AJ, 106, 1826

Howard, C. D., Rich, R. M., Reitzel, D. B., et al. 2008, ApJ, 688, 1060

Howard, C. D., Rich, R. M., Clarkson, W., et al. 2009, ApJ, 702, L153

Immeli, A., Samland, M., Gerhard, O., \& Westera, P. 2004, A\&A, 413, 547

Johnson, J. A., Dong, S., \& Gould, A. 2009, ApJ, submitted, [arXiv:0910.3670v1]

Johnson, J. A., Gal-Yam, A., Leonard, D. C., et al. 2007, ApJ, 655, L33

Johnson, J. A., Gaudi, B. S., Sumi, T., Bond, I. A., \& Gould, A. 2008, ApJ, 685, 508

Kalirai, J. S., Bergeron, P., Hansen, B. M. S., et al. 2007, ApJ, 671, 748

Kane, S. R., \& Sahu, K. C. 2006, ApJ, 637, 752

Kilic, M., Stanek, K. Z., \& Pinsonneault, M. H. 2007, ApJ, 671, 761

Kormendy, J., \& Kennicutt, R. C. 2004, ARA\&A, 42, 603

Lecureur, A., Hill, V., Zoccali, M., et al. 2007, A\&A, 465, 799

Luck, R. E., \& Heiter, U. 2007, AJ, 133, 2464

Maeder, A. 1992, A\&A, 264, 105

Matteucci, F. 2001, The chemical evolution of the Galaxy (Dordrecht: Kluwer Academic Publishers), ApS\&S library, 253

McWilliam, A., \& Rich, R. M. 1994, ApJS, 91, 749

McWilliam, A., Matteucci, F., Ballero, S., et al. 2008, AJ, 136, 367

Meléndez, J., Asplund, M., Alves-Brito, A., et al. 2008, A\&A, 484, L21

Meléndez, J., Casagrande, L., Ramírez, I., \& Asplund, M. 2009, A\&A, submitted Meynet, G., \& Maeder, A. 2002, A\&A, 390, 561

Minniti, D., \& Zoccali, M. 2008, in IAU Symp. 245, ed. M. Bureau, E. Athanassoula, \& B. Barbuy, 323

Minniti, D., Vandehei, T., Cook, K. H., Griest, K., \& Alcock, C. 1998, ApJ, 499, L175

Nair, V., \& Miralda-Escudé, J. 1999, ApJ, 515, 206

Nordström, B., Mayor, M., Andersen, J., et al. 2004, A\&A, 418, 989

Oort, J. H. 1977, ARA\&A, 15, 295

Prochaska, J. X., Naumov, S. O., Carney, B. W., McWilliam, A., \& Wolfe, A. M. 2000, AJ, 120, 2513

Rahimi, A., Kawata, D., Brook, C. B., \& Gibson, B. K. 2010, MNRAS, 401, 1826

Ramírez, I., \& Meléndez, J. 2005, ApJ, 626, 465

Rattenbury, N. J., Mao, S., Sumi, T., \& Smith, M. C. 2007, MNRAS, 378, 1064 Rich, R. M., \& Origlia, L. 2005, ApJ, 634, 1293

Rich, R. M., Origlia, L., \& Valenti, E. 2007, ApJ, 665, L119

Ryde, N., Gustafsson, B., Edvardsson, B., et al. 2010, A\&A, 509, A20

Sahu, K. C., Casertano, S., Bond, H. E., et al. 2006, Nature, 443, 534

Santos, N. C., Lovis, C., Pace, G., Melendez, J., \& Naef, D. 2009, A\&A, 493, 309

Schönrich, R., \& Binney, J. 2009, MNRAS, 396, 203

Shi, J. R., Gehren, T., \& Zhao, G. 2004, A\&A, 423, 683

Sofue, Y., Honma, M., \& Omodaka, T. 2009, PASJ, 61, 227

Takeda, Y., Sato, B., \& Murata, D. 2008, PASJ, 60, 781

Taylor, B. J., \& Croxall, K. 2005, MNRAS, 357, 967

Twarog, B. A. 1980, ApJ, 242, 242

Udalski, A. 2003, Acta Astron., 53, 291

Venn, K. A., Irwin, M., Shetrone, M. D., et al. 2004, AJ, 128, 1177

Weiland, J. L., Arendt, R. G., Berriman, G. B., et al. 1994, ApJ, 425, L81

Woosley, S. E., \& Weaver, T. A. 1995, ApJS, 101, 181

Yoo, J., DePoy, D. L., Gal-Yam, A., et al. 2004, ApJ, 603, 139

Zoccali, M., Renzini, A., Ortolani, S., et al. 2003, A\&A, 399, 931

Zoccali, M., Lecureur, A., Barbuy, B., et al. 2006, A\&A, 457, L1

Zoccali, M., Hill, V., Lecureur, A., et al. 2008, A\&A, 486, 177 